\title{
Platinum(II)-Mediated Coupling Reactions of Acetonitrile with the Exocyclic Nitrogen of 9-Methyladenine and 1-Methylcytosine. Synthesis, NMR Characterization, and X-ray Structures of New Azametallacycle Complexes
}

\author{
Bruno Longato, ${ }^{*, \dagger \neq}$ Diego Montagner, ${ }^{\ddagger}$ Giuliano Bandoli, ${ }^{\S}$ and Ennio Zangrando ${ }^{\perp}$ \\ Istituto di Scienze e Tecnologie Molecolari-C.N.R., c/o Dipartimento di Scienze Chimiche, \\ Universita' di Padova, Via Marzolo 1, 35131-Padova, Italy, Dipartimento di Scienze \\ Farmaceutiche, Universita' di Padova, Via Marzolo 5, 35131-Padova, Italy, and \\ Dipartimento di Scienze Chimiche, Universita' di Trieste, Via Giorgieri 1, 34127 Trieste, Italy \\ Received October 10, 2005
}

The hydroxo complex cis- $\left[\mathrm{L}_{2} \mathrm{Pt}(\mu-\mathrm{OH})\right]_{2}\left(\mathrm{NO}_{3}\right)_{2},\left(\mathrm{~L}=\mathrm{PMePh}_{2}, 1 \mathrm{a}\right)$, in $\mathrm{CH}_{3} \mathrm{CN}$ solution, deprotonates the $\mathrm{NH}_{2}$ group of 9-methyladenine (9-MeAd) to give the cyclic trinuclear species cis- $\left[\mathrm{L}_{2} \mathrm{Pt}\{9 \mathrm{~g}-\mathrm{MeAd}(-\mathrm{H})\}\right]_{3}\left(\mathrm{NO}_{3}\right)_{3},\left(\mathrm{~L}=\mathrm{PMePh}_{2}\right.$, 2a), in which the nucleobase binds the metal centers through the $N(1), N(6)$ atoms. In solution at room temperature, 2a slowly reacts with the solvent to form quantitatively the mononuclear azametallacycle cis- $\left[\mathrm{L}_{2} \mathrm{PtNH}=\mathrm{C}(\mathrm{Me})\{9\right.$ $\operatorname{MeAd}(-2 \mathrm{H})\}] \mathrm{NO}_{3}\left(\mathrm{~L}=\mathrm{PMePh}_{2}\right.$, 3a), containing as anionic ligand the deprotonated form of molecule $\mathrm{N}$-(9-methyl1,9-dihydro-purin-6-ylidene)-acetamidine. In the same experimental conditions, the hydroxo complex with $\mathrm{PPh}_{3}(\mathbf{1 b})$ forms immediately the insertion product $3 \mathrm{~b}$. Single-crystal $\mathrm{X}$-ray analyses of $\mathbf{3 a}$ and $\mathbf{3 b}$ show the coordination of the platinum cation at the $\mathrm{N}(1)$ site of the purine moiety and to the $\mathrm{N}$ atom of the inserted acetonitrile, whereas the exocyclic amino nitrogen binds the carbon atom of the same $\mathrm{CN}$ group. The resulting six-membered ring is slightly distorted from planarity, with carbon-nitrogen bond distances for the inserted nitrile typical of a double bond [C(3)$\mathrm{N}(2)=1.292(7) \AA$ in $3 \mathrm{a}$ and $1.279(11) \AA$ in $3 \mathbf{b}$ ], while the remaining $\mathrm{CN}$ bonds of the metallocycle are in the range of $1.335(8)-1.397(10) \AA$. A detailed multinuclear ${ }^{1} \mathrm{H},{ }^{31} \mathrm{P},{ }^{13} \mathrm{C}$, and ${ }^{15} \mathrm{~N}$ NMR study shows that the nitrogen atom of the inserted acetonitrile molecule binds a proton suggesting for $\mathbf{3 a}, \mathbf{b}$ an imino structure in solution. In DMSO and chlorinated solvents, $3 a$ slowly releases the nitrile reforming the trinuclear species $2 \mathbf{a}$, whereas $3 \mathbf{b}$ forms the mononuclear derivative cis- $\left[\mathrm{L}_{2} \mathrm{Pt}\{9-\mathrm{MeAd}(-\mathrm{H})\}\right] \mathrm{NO}_{3}\left(\mathrm{~L}=\mathrm{PPh}_{3}, \mathbf{4 b}\right)$, in which the adeninate ion chelates the metal center through the $\mathrm{N}(6)$ and $\mathrm{N}(7)$ atoms. Complex $4 \mathrm{~b}$ is quantitatively obtained when $1 \mathrm{~b}$ reacts with 9 -MeAd in DMSO and can be easily isolated if the reaction is carried out in $\mathrm{CH}_{2} \mathrm{Cl}_{2}$. In $\mathrm{CH}_{3} \mathrm{CN}$ solution, at room temperature, $4 \mathrm{~b}$ slowly converts into $3 \mathrm{~b}$ indicating that the insertion of acetonitrile is a reversible process. A similar metalmediated coupling reaction occurs when 1a,b react with 1-methylcytosine (1-MeCy) in $\mathrm{CH}_{3} \mathrm{CN}$. The resulting complexes, cis- $\left[\mathrm{L}_{2} \mathrm{PtNH}=\mathrm{C}(\mathrm{Me})\{1-\mathrm{MeCy}(-2 \mathrm{H})\}\right] \mathrm{NO}_{3},\left(\mathrm{~L}=\mathrm{PMePh}_{2}, 5 \mathrm{a}\right.$ and $\left.\mathrm{PPh}_{3}, 5 \mathbf{b}\right)$, contain the deprotonated form of the ligand $\mathrm{N}$-(1-methyl-2-oxo-2,3-dihydro-1 H-pyrimidin-4-ylidene)-acetamidine. The $\mathrm{X}$-ray analysis of 5a shows the coordination of the metal at the $\mathrm{N}(3)$ site of the pyrimidine cycle and to the nitrogen atom of the acetonitrile, with features of the six-membered metallocycle only slightly different from those found in $3 a$ and $3 b$. In $\mathrm{CD}_{3} \mathrm{CN} /$ $\mathrm{CH}_{3}{ }^{13} \mathrm{CN}$ solution complexes $5 \mathbf{a}, \mathbf{b}$ undergo exchange of the inserted nitrile, while in DMSO or chlorinated solvents they irreversibly release $\mathrm{CH}_{3} \mathrm{CN}$ to form species not yet fully characterized. No insertion of $\mathrm{CH}_{3} \mathrm{CN}$ occurs when the hydroxo complexes are stabilized by $\mathrm{PMe}_{3}$ and $\mathrm{PMe}_{2} \mathrm{Ph}$.

\section{Introduction}

The chemistry of metal-activated organonitriles $(\mathrm{RCN})$ is still intensively investigated owing to the wide variety of products obtainable by addition reactions to the $\mathrm{C} \equiv \mathrm{N}$

\footnotetext{
* To whom correspondence should be addressed. E-mail: longato@chin.unipd.it.

'Istituto di Scienze e Tecnologie Molecolari, Universita’ di Padova.

$\doteqdot$ Dipartimento di Scienze Chimiche, Universita’ di Padova.

$\S$ Dipartimento di Scienze Farmaceutiche, Universita’ di Padova.

$\perp$ Universita' di Trieste.
}

functionality. ${ }^{1}$ It is now clear that the coordination of $\mathrm{RC} \equiv$ $\mathrm{N}$ to a metal center $[\mathrm{M}]$ increases the rate of the nucleophilic attack to the carbon atom of the $\mathrm{CN}$ group. Depending on the nature of the nucleophile $(\mathrm{Nu})$ - protic or aprotic-the product is the imino derivative, $[\mathrm{M}]-\mathrm{NH}=\mathrm{C}(\mathrm{Nu}) \mathrm{R}$, or the

(1) (a) Kukushkin, V. Yu.; Pombeiro, A. J. L. Chem. Rev. 2002, 102 1771-1802. (b) Michelin, R. A.; Mozzon, M.; Bertani, R. Coord. Chem. Rev. 1996, 147, 299-338. (c) Reisner, E.; Arion, V. B.; Chioresku, J.; Schmid, W. F. J. Chem. Soc., Dalton Trans. 2005, $2355-2364$

Inorganic Chemistry, Vol. 45, No. 4, 20061805 
Chart 1<smiles>CN(C)c1ncnc2c1ncn2C</smiles>

9-MeAd<smiles>Cn1ccc(N)nc1=O</smiles>

1-MeCy azavinyledene species $[\mathrm{M}]-\mathrm{N}=\mathrm{C}(\mathrm{Nu}) \mathrm{R}$, respectively. As an example of the first type of reaction, we have recently reported the characterization of the amidine complex cis$\left[\left(\mathrm{PMe}_{3}\right)_{2} \mathrm{Pt}\left\{\mathrm{NH}=\mathrm{C}(\mathrm{Me}) \mathrm{NH}_{2}\right\}\{1-\mathrm{MeTy}(-\mathrm{H})\}\right]^{+}$in which $1-\operatorname{MeTy}(-\mathrm{H})$ is the anion of the model nucleobase 1-methylthymine, metal coordinated at the N(3) site. The compound was obtained in low yield from the cationic species cis- $\left[\left(\mathrm{PMe}_{3}\right)_{2} \mathrm{Pt}(\mathrm{N} \equiv \mathrm{CMe})\{1-\mathrm{MeTy}(-\mathrm{H})\}\right]^{+}$, left in acetonitrile containing stoichiometric amounts of water. ${ }^{2}$

Azavinyledene complexes can be obtained by formal addition of a metal-ligand fragment to a nitrile triple bond. Insertion of acetonitrile into metal-nitrogen bonds of early transition metal amides, $\mathrm{M}-\mathrm{NR}_{2}$, to form the adducts $[\mathrm{M}]-\mathrm{N}=\mathrm{C}(\mathrm{Me}) \mathrm{NR}_{2}$ is well documented $\left(\mathrm{M}=\mathrm{Ta}^{3}\right)$, and a similar reaction occurs with $\mathrm{M}-\mathrm{PR}_{2}$ bonds $(\mathrm{M}=\mathrm{Zr})$, generating phosphorus analogues of $N, N$-dialkylamidinates. ${ }^{4}$

In this Article we report four examples of insertion of acetonitrile into platinum-nitrogen bonds of the $\mathrm{NH}_{2}$ deprotonated nucleobases 9-methyadenine (9-MeAd) and 1-methylcytosine (1-MeCy), depicted in Chart 1, observed in the reactions of the hydroxo complexes $c i s-\left[\mathrm{L}_{2} \mathrm{Pt}(\mu-\mathrm{OH})\right]_{2}-$ $\left(\mathrm{NO}_{3}\right)_{2}\left(\mathrm{~L}=\mathrm{PMePh}_{2}, \mathrm{PPh}_{3}\right)$ with the model nucleobases in $\mathrm{CH}_{3} \mathrm{CN}$ solution.

The structures of the new complexes have been elucidated by single-crystal X-ray analysis and multinuclear NMR spectroscopy showing that in solution they can be formulated as mononuclear azametallacycle species, cis- $\left[\mathrm{L}_{2} \mathrm{PtNH}=\right.$ $\mathrm{C}(\mathrm{Me})\{9-\mathrm{MeAd}(-2 \mathrm{H})\}]^{+}$and $c i s-\left[\mathrm{L}_{2} \mathrm{PtNH}=\mathrm{C}(\mathrm{Me})\{1-\mathrm{MeCy}-\right.$ $(-2 \mathrm{H})\}]^{+}\left(\mathrm{L}=\mathrm{PMePh}_{2}, \mathrm{PPh}_{3}\right)$, in which the anionic ligands are the deprotonated forms of the amidines $N$-(9-methyl1,9-dihydro-purin-6-ylidene)-acetamidine and $N$-(1-methyl2-oxo-2,3-dihydro-1H-pyrimidin-4-ylidene)-acetamidine, respectively, shown in Chart 2.

The stability in solution of this new class of metallocycles has been also investigated showing that $c i s-\left[\mathrm{L}_{2} \mathrm{PtNH}=\right.$ $\mathrm{C}(\mathrm{Me})\{9-\mathrm{MeAd}(-2 \mathrm{H})\}]^{+}$in chlorinated solvents release reversibly the inserted $\mathrm{CH}_{3} \mathrm{CN}$ molecule to form the cyclic species cis- $\left[\mathrm{L}_{2} \mathrm{Pt}\{9-\mathrm{MeAd}(-\mathrm{H})\}\right]_{n}{ }^{n+}$ in which binding modes of the deprotonated nucleobase and nuclearity of resulting cations depend on the nature of L. ${ }^{5}$ To the best of our

(2) Longato, B.; Bandoli, G.; Mucci, A.; Schenetti, L. Eur. J. Inorg. Chem. 2001, 3021-3029.

(3) Broder, C. K.; Goeta, A. E.; Howard, A. K.; Hughes, A. K.; Johnson, A. L.; Malget, J. M.; Wade, K. J. Chem. Soc., Dalton Trans. 2000, 3526-3533.

(4) Segerer, U.; Blaurock, S.; Sieler, J.; Hey-Hawkins, E. Organometallics 1999, 18, 2838-2842.

(5) Longato, B.; Pasquato, L.; Mucci, A.; Schenetti, L.; Zangrando, E. Inorg. Chem. 2003, 42, 7861-7871.
Chart 2

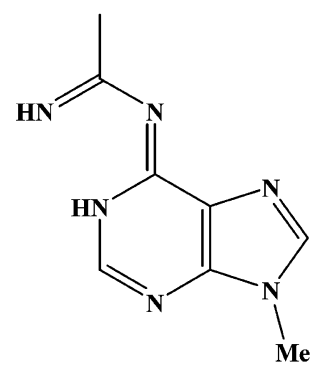

$N$-(9-Methyl-1,9-dihydro-purin6-ylidene)-acetamidine<smiles>CC(=N)N=c1ccn(C#N)c(=O)[nH]1</smiles>

$N$-(1-Methyl-2-oxo-2,3-dihydro$1 H$-pyrimidin-4-ylidene)-acetamidine knowledge, the reversible insertion of $\mathrm{CH}_{3} \mathrm{CN}$ into a metalnitrogen bond has been reported only in some tetranuclear $\mathrm{Ir}_{2} \mathrm{Ag}_{2}$ pyrazolyl amidine complexes. ${ }^{6}$

Moreover, in this Article we show that the insertion of $\mathrm{CH}_{3} \mathrm{CN}$ does not occur in platinum nucleobase compounds stabilized by $\mathrm{PMe}_{3}$ and $\mathrm{PMe}_{2} \mathrm{Ph}$ ligands. It turns out that the adenine complexes cis-[(PMe $\left.)_{2} \mathrm{Pt}\{9-\mathrm{MeAd}(-\mathrm{H})\}\right]_{2}{ }^{2+}$ and cis- $\left[\left(\mathrm{PMe}_{2} \mathrm{Ph}\right)_{2} \mathrm{Pt}\{9-\mathrm{MeAd}(-\mathrm{H})\}\right]_{3}{ }^{3+}$, previously characterized, ${ }^{7,8}$ can be prepared in acetonitrile and are indefinitely stable in this solvent, even at high temperature. Similarly, in the reaction of cis- $\left[\left(\mathrm{PMe}_{2} \mathrm{Ph}\right)_{2} \mathrm{Pt}(\mu-\mathrm{OH})\right]_{2}\left(\mathrm{NO}_{3}\right)_{2}$, with 1-MeCy in $\mathrm{CH}_{3} \mathrm{CN}$, the deprotonation of the nucleobase affords the trinuclear species cis- $\left[\left(\mathrm{PMe}_{2} \mathrm{Ph}\right)_{2} \mathrm{Pt}\{1-\mathrm{MeCy}(-\right.$ $\mathrm{H})\}]_{3}\left(\mathrm{NO}_{3}\right)_{3}$ which does not react further with the solvent. Insertion reactions of the acetonitrile $\mathrm{C} \equiv \mathrm{N}$ group into metalnucleobase bonds have been previously reported for a rhenium (IV)-adenine complex. ${ }^{9}$

\section{Experimental Section}

Instrumentation and Materials. ${ }^{1} \mathrm{H},{ }^{13} \mathrm{C}$, and ${ }^{31} \mathrm{P}$ NMR spectra were recorded on a Bruker AVANCE 300 spectrometer (at 300.13, 75.47, and $121.49 \mathrm{MHz}$, respectively), equipped with a variable temperature apparatus and were calibrated against the residual signals of the solvent (for ${ }^{1} \mathrm{H}$ and ${ }^{13} \mathrm{C}$ ) and external $\mathrm{H}_{3} \mathrm{PO}_{4}$ for ${ }^{31} \mathrm{P}$. ${ }^{1} \mathrm{H},{ }^{15} \mathrm{~N}$ heterocorrelation experiments were performed on a Bruker $400 \mathrm{MHz}$ spectrometer, and ${ }^{15} \mathrm{~N}$ resonances were calibrated with nitromethane. The solvents $\mathrm{CD}_{2} \mathrm{Cl}_{2}, \mathrm{CD}_{3} \mathrm{CN}$, and $\mathrm{CH}_{3}{ }^{13} \mathrm{CN}$ (Aldrich) were distilled from $\mathrm{CaH}_{2}$.

Reagent grade chemicals were used as received unless otherwise stated. cis- $\left[\left(\mathrm{PMe}_{3}\right)_{2} \mathrm{Pt}(\mu-\mathrm{OH})\right]_{2}\left(\mathrm{ClO}_{4}\right)_{2},{ }^{2}$ (Caution: Perchlorates are potential explosives! $)$ cis- $\left[\left(\mathrm{PMe}_{2} \mathrm{Ph}\right)_{2} \mathrm{Pt}(\mu-\mathrm{OH})\right]_{2}\left(\mathrm{NO}_{3}\right)_{2},{ }^{7}$ cis$\left[\left(\mathrm{PMePh}_{2}\right)_{2} \mathrm{Pt}(\mu-\mathrm{OH})\right]_{2}\left(\mathrm{NO}_{3}\right)_{2},{ }^{10}$ and $9-\mathrm{MeAd}^{11}$ were synthesized as previously reported.

Synthetic Work. 1. cis- $\left[\left(\mathrm{PPh}_{3}\right)_{2} \mathbf{P t}(\mu-\mathrm{OH})\right]_{2}\left(\mathrm{NO}_{3}\right)_{2}$ (1b). The complex was prepared following the procedure described for the synthesis of the $\mathrm{PMePh}_{2}$ analogue, with a yield of $69 \% .^{10}$ Elemental Anal. Calcd for $\mathrm{C}_{36} \mathrm{H}_{31} \mathrm{NO}_{4} \mathrm{P}_{2} \mathrm{Pt}$ : C, 54.14; H, 3.91; N, 1.75 .

(6) Carmona, D.; Ferrer, J.; Lahoz, F. J.; Oro, L. A.; Lamata, M. P. Organometallics 1996, 15 (5), 5175-5178.

(7) Schenetti, L.; Mucci, A.; Longato, B. J. Chem. Soc., Dalton Trans. 1996, 299-303.

(8) Longato, B.; Pasquato, L.; Mucci, A.; Schenetti, L. Eur. J. Inorg Chem. 2003, 128-137.

(9) Pearson, C.; Beauchamp, A. L. Inorg. Chem. 1998, 37, 1242-1248.

(10) Longato, B.; Bandoli, G.; Dolmella, A. Eur. J. Inorg. Chem. 2004, 1092-1099.

(11) Talman, E. G.; Brüning, W; Reedijk, J.; Spek, A. L.; Veldman, N. Inorg. Chem. 1997, 36, 854-861. 
Table 1. Crystal Data and Details of Refinements for Compounds 3a, $3 \mathbf{b}$, and $5 \mathbf{a}$

\begin{tabular}{|c|c|c|c|}
\hline & 3a & 3b & $5 \mathbf{a}$ \\
\hline formula & $\mathrm{C}_{34} \mathrm{H}_{35} \mathrm{~N}_{7} \mathrm{O}_{3} \mathrm{P}_{2} \mathrm{Pt}$ & $\mathrm{C}_{44} \mathrm{H}_{39} \mathrm{~N}_{7} \mathrm{O}_{3} \mathrm{P}_{2} \mathrm{Pt}$ & $\mathrm{C}_{33} \mathrm{H}_{35} \mathrm{~N}_{5} \mathrm{O}_{4} \mathrm{P}_{2} \mathrm{P}$ \\
\hline & 846.72 & 970.85 & 822.69 \\
\hline crystal syst & triclinic & triclinic & orthorhombic \\
\hline space group & $P \overline{1}$ & $P \overline{1}$ & $P n a 2_{1}$ \\
\hline$a(\AA)$ & $10.236(2)$ & $9.741(2)$ & $14.933(4)$ \\
\hline$b(\AA)$ & $11.563(2)$ & $12.303(3)$ & $18.888(4)$ \\
\hline$c(\AA)$ & $16.510(3)$ & $17.180(3)$ & $11.860(3)$ \\
\hline$\alpha\left({ }^{\circ}\right)$ & $107.79(3)$ & $95.95(3)$ & 90.0 \\
\hline$\beta\left(^{\circ}\right)$ & $93.34(3)$ & $96.78(3)$ & 90.0 \\
\hline$\gamma\left({ }^{\circ}\right)$ & $110.22(3)$ & $105.30(3)$ & 90.0 \\
\hline $\operatorname{vol}\left(\AA^{3}\right)$ & $1716.40(6)$ & $1952.4(7)$ & $3345.2(14)$ \\
\hline $\mathrm{Z}$ & 2 & 2 & 4 \\
\hline$D_{\text {calcd }}\left(\mathrm{g} \mathrm{cm}^{-3}\right)$ & 1.638 & 1.651 & 1.634 \\
\hline$\lambda \operatorname{Mo~K} \alpha\left(\mathrm{mm}^{-1}\right)$ & 4.226 & 3.727 & 4.335 \\
\hline$F(000)$ & 840 & 968 & 1632 \\
\hline unique reflns & 6014 & 8422 & 9157 \\
\hline reflns $I>2 \sigma(I)$ & 4370 & 3614 & 6823 \\
\hline refined params & 424 & 512 & 406 \\
\hline GOF & 0.903 & 0.965 & 0.906 \\
\hline Flack param & & & $0.016(6)$ \\
\hline $\mathrm{R} 1(I>2 \sigma(I))^{a}$ & 0.0398 & 0.0455 & 0.0322 \\
\hline $\mathrm{wR} 2^{b}$ & 0.0667 & 0.0652 & 0.0667 \\
\hline max residuals $\left(\mathrm{e} / \AA^{3}\right)$ & 1.017 & 0.673 & 0.476 \\
\hline
\end{tabular}

${ }^{a} \mathrm{R} 1=\sum|| F_{\mathrm{o}}|-| F_{\mathrm{c}}|| / \sum\left|F_{\mathrm{o}}\right| .{ }^{b} \mathrm{wR} 2=\left[\sum w\left(F_{\mathrm{o}}{ }^{2}-F_{\mathrm{c}}{ }^{2}\right)^{2} / \sum w\left(F_{\mathrm{o}}{ }^{2}\right)^{2}\right]^{1 / 2}$.

Found: $\mathrm{C}, 53.69 ; \mathrm{H}, 3.84 ; \mathrm{N}, 1.82 .{ }^{1} \mathrm{H}$ NMR in $\mathrm{CDCl}_{3}$ : 2.12 (br $\mathrm{s}, 1 \mathrm{H}, \mathrm{OH}) ; 7.55-7.11(\mathrm{~cm}, 30 \mathrm{H}, \mathrm{Ph}) .\left\{{ }^{1} \mathrm{H}\right\}^{31} \mathrm{P} \mathrm{NMR}$ in $\mathrm{CDCl}_{3}$ : singlet at $\delta 8.45\left({ }^{1} J_{\mathrm{PPt}}=3713 \mathrm{~Hz}\right)$. These spectroscopic data compare well with those of the $\mathrm{BF}_{4}$ analogue. ${ }^{12}$

2. $\operatorname{cis}-\left[\left(\mathrm{PMePh}_{2}\right)_{2} \mathrm{PtNH}=\mathrm{C}(\mathrm{Me})\{\mathbf{9}-\mathrm{MeAd}(-2 \mathrm{H})\}\right] \mathrm{NO}_{3}(3 \mathrm{a}) . \mathrm{A}$ suspension of cis- $\left[\left(\mathrm{PMePh}_{2}\right)_{2} \mathrm{Pt}(\mu-\mathrm{OH})\right]_{2}\left(\mathrm{NO}_{3}\right)_{2}, \mathbf{1 a},(716 \mathrm{mg}, 0.5$ mmol) and 9-MeAd (158 mg, $1.1 \mathrm{mmol})$ in $\mathrm{CH}_{3} \mathrm{CN}(26 \mathrm{~mL})$ was stirred for ca. $1 \mathrm{~h}$, and the resulting pale yellow solution was heated at $50{ }^{\circ} \mathrm{C}$ for $12 \mathrm{~h}$. A trace amount of metallic platinum was removed by filtration, and the solution was left to evaporate at room temperature. In 2-3 days pale yellow crystals, suitable for the X-ray analysis, were formed, which were separated from the solution and dried under vacuum (ca. $20 \mathrm{mg})$. Addition of $\mathrm{Et}_{2} \mathrm{O}(25 \mathrm{~mL})$ to the remaining solution caused the precipitation of a very pale yellow solid which was collected by filtration, washed with $\mathrm{Et}_{2} \mathrm{O}$, and dried under vacuum. The recovered solid $(600 \mathrm{mg})$ was purified by dissolution in $\mathrm{CH}_{3} \mathrm{CN}$ and precipitated by addition of diethyl ether. The yield of pure $\mathbf{3 a}$ (pale yellow microcrystals) was $69 \%$. Elemental Anal. Calcd for $\mathrm{C}_{34} \mathrm{H}_{35} \mathrm{~N}_{7} \mathrm{O}_{3} \mathrm{P}_{2} \mathrm{Pt}$ : C, 48.23; H, 4.17; N, 11.58. Found: C, 48.20; H, 4.10; N, 11.48. ${ }^{1} \mathrm{H}$ and ${ }^{15} \mathrm{~N}$ NMR data are collected in Tables 3 and 4 , respectively. $\left\{{ }^{1} \mathrm{H}\right\}{ }^{31} \mathrm{P}$ NMR in $\mathrm{CD}_{3^{-}}$ $\mathrm{CN}$ : $\mathrm{AB}$ multiplet at $\delta-3.36\left({ }^{1} J_{\mathrm{PPt}}=3172 \mathrm{~Hz}\right)$ and $-4.01\left({ }^{1} J_{\mathrm{PPt}}\right.$ $=3265 \mathrm{~Hz}$ ) with ${ }^{2} J_{\mathrm{PP}}=27.4 \mathrm{~Hz} .\left\{{ }^{1} \mathrm{H}\right\}{ }^{13} \mathrm{C} \mathrm{NMR}$ (in $\left.\mathrm{CD}_{3} \mathrm{CN}\right)$ : (9MeAd resonances) $156.18\left(\mathrm{~d},{ }^{3} J_{\mathrm{CP}}=8.5 \mathrm{~Hz}, \mathrm{C}-2\right), 150.42$ (s, C-6), 149.56 (s, C-4), 144.16 (s, C-8), 30.05 (s, $\left.\mathrm{NCH}_{3}\right) . \mathrm{PMePh}_{2}$ resonances: $133.24\left(\mathrm{~d},{ }^{3} J_{\mathrm{CP}}=10.3 \mathrm{~Hz}, \mathrm{C}-2\right.$ and C-6), 133.05 (d, $\left.{ }^{2} J_{\mathrm{CP}}=10.7 \mathrm{~Hz}, \mathrm{C}-2^{\prime}, \mathrm{C}-6{ }^{\prime}\right), 132.89\left(\mathrm{~d},{ }^{4} J_{\mathrm{CP}}=2.5 \mathrm{~Hz}, \mathrm{C}-4\right), 132.11$ $\left(\mathrm{d},{ }^{4} J_{\mathrm{CP}}=2.3 \mathrm{~Hz}, \mathrm{C}-4\right), 129.84\left(\mathrm{~d},{ }^{3} J_{\mathrm{CP}}=11.1 \mathrm{~Hz}, \mathrm{C}-3\right.$ and C-5), $129.54\left(\mathrm{~d}, J_{\mathrm{CP}},=10.8 \mathrm{~Hz}, \mathrm{C}-3\right.$ and C-5), $128.47\left(\mathrm{dd}, J_{\mathrm{CP}}=60.0\right.$ and $3.5 \mathrm{~Hz}, \mathrm{C}-1), 126.80\left(\mathrm{dd}, J_{\mathrm{CP}}=63.3\right.$ and $\left.3.5 \mathrm{~Hz}, \mathrm{C}-1\right), 14.41$ (dd, $J_{\mathrm{CP}}=44.4$ and $\left.3.5 \mathrm{~Hz}, \mathrm{PCH}_{3}\right), 12.82\left(\mathrm{dd},{ }^{1} J_{\mathrm{CP}}=45.8\right.$ and 3.7 $\left.\mathrm{Hz}, \mathrm{PCH}_{3}\right) . \mathrm{CH}_{3} \mathrm{CN}$ resonances: $164.96\left(\mathrm{~s},{ }^{2} J_{\mathrm{CPt}}=12 \mathrm{~Hz}\right) .\left\{{ }^{1} \mathrm{H}\right\}^{31} \mathrm{P}$ $\mathrm{NMR}$ in $\mathrm{CDCl}_{3}$ : $\mathrm{AB}$ multiplet at $\delta-3.21\left({ }^{1} J_{\mathrm{PPt}}=3173 \mathrm{~Hz}\right)$ and $-3.50\left({ }^{1} J_{\mathrm{PPt}}=3215 \mathrm{~Hz}\right)$ with ${ }^{2} J_{\mathrm{PP}}=27.4 \mathrm{~Hz} .\left\{{ }^{1} \mathrm{H}\right\}{ }^{31} \mathrm{P} \mathrm{NMR}$ in

(12) (a) Bushnell, G. W.; Dixon, K. R.; Hunter, R. G.; McFarland, J. J. Can. J. Chem. 1972, 50, 3694-3699. (b) Li, J. J.; Li, W.; James, A. J.; Holbert, T.; Sharp, T.; Sharp, P. R. Inorg. Chem. 1999, 38, 15631572.
Table 2. Selected Bond Lengths ( $\AA$ ) and Angles (Deg) in the Cation of $\mathbf{3 a}$ and $\mathbf{3 b}$

\begin{tabular}{lll}
\hline & $\mathbf{3 a}$ & $\mathbf{3 b}$ \\
\hline $\mathrm{Pt}-\mathrm{N}(1)$ & $2.115(5)$ & $2.124(7)$ \\
$\mathrm{Pt}-\mathrm{N}(2)$ & $2.017(5)$ & $2.011(7)$ \\
$\mathrm{Pt}-\mathrm{P}(1)$ & $2.290(2)$ & $2.311(3)$ \\
$\mathrm{Pt}-\mathrm{P}(2)$ & $2.267(2)$ & $2.281(3)$ \\
$\mathrm{N}(2)-\mathrm{C}(3)$ & $1.292(7)$ & $1.279(11)$ \\
$\mathrm{C}(3)-\mathrm{C}(7)$ & $1.523(9)$ & $1.509(11)$ \\
$\mathrm{N}(6)-\mathrm{C}(3)$ & $1.335(8)$ & $1.358(11)$ \\
$\mathrm{N}(6)-\mathrm{C}(6)$ & $1.355(8)$ & $1.319(9)$ \\
$\mathrm{N}(1)-\mathrm{C}(2)$ & $1.365(8)$ & $1.349(9)$ \\
$\mathrm{N}(1)-\mathrm{C}(6)$ & $1.357(7)$ & $1.397(10)$ \\
$\mathrm{N}(1)-\mathrm{Pt}-\mathrm{N}(2)$ & $85.1(2)$ & $84.4(3)$ \\
$\mathrm{N}(1)-\mathrm{Pt}-\mathrm{P}(1)$ & $95.08(15)$ & $93.04(19)$ \\
$\mathrm{N}(1)-\mathrm{Pt}-\mathrm{P}(2)$ & $172.31(15)$ & $173.5(2)$ \\
$\mathrm{N}(2)-\mathrm{Pt}-\mathrm{P}(1)$ & $172.43(18)$ & $176.3(3)$ \\
$\mathrm{N}(2)-\mathrm{Pt}-\mathrm{P}(2)$ & $88.17(16)$ & $89.4(2)$ \\
$\mathrm{P}(1)-\mathrm{Pt}-\mathrm{P}(2)$ & $92.05(7)$ & $93.08(10)$ \\
$\mathrm{C}(6)-\mathrm{N}(1)-\mathrm{C}(2)$ & $118.6(6)$ & $119.8(8)$ \\
$\mathrm{C}(6)-\mathrm{N}(1)-\mathrm{Pt}$ & $122.5(4)$ & $119.1(5)$ \\
$\mathrm{C}(2)-\mathrm{N}(1)-\mathrm{Pt}$ & $118.9(4)$ & $120.1(6)$ \\
$\mathrm{C}(3)-\mathrm{N}(2)-\mathrm{Pt}$ & $127.1(5)$ & $128.6(7)$ \\
$\mathrm{N}(2)-\mathrm{C}(3)-\mathrm{N}(6)$ & $128.0(6)$ & $126.5(8)$ \\
$\mathrm{N}(2)-\mathrm{C}(3)-\mathrm{C}(7)$ & $117.3(6)$ & $119.6(9)$ \\
$\mathrm{N}(6)-\mathrm{C}(3)-\mathrm{C}(7)$ & $114.7(6)$ & $113.9(8)$ \\
$\mathrm{C}(3)-\mathrm{N}(6)-\mathrm{C}(6)$ & $122.4(6)$ & $123.1(8)$ \\
$\mathrm{N}(1)-\mathrm{C}(6)-\mathrm{N}(6)$ & $127.3(6)$ & $127.7(8)$ \\
$\mathrm{N}(1)-\mathrm{C}(6)-\mathrm{C}(5)$ & $116.1(6)$ & $113.5(8)$ \\
$\mathrm{N}(6)-\mathrm{C}(6)-\mathrm{C}(5)$ & $116.6(6)$ & $118.7(8)$ \\
& &
\end{tabular}

DMSO- $d_{6}:$ AB multiplet at $\delta-3.05\left({ }^{1} J_{\mathrm{PPt}}=3182 \mathrm{~Hz}\right)$ and -3.62 $\left({ }^{1} J_{\mathrm{PPt}}=3264 \mathrm{~Hz}\right)$ with ${ }^{2} J_{\mathrm{PP}}=26.8 \mathrm{~Hz}$.

3a, dissolved in a mixture of $\mathrm{CD}_{3} \mathrm{CN}$ and $\mathrm{CH}_{3}{ }^{13} \mathrm{CN}(2: 1 \mathrm{v} / \mathrm{v})$, exchanges the inserted $\mathrm{CH}_{3} \mathrm{CN}$ molecule in a few hours at room temperature, as shown by the appearance of a ${ }^{13} \mathrm{C}$ resonance at $164.3 \mathrm{ppm}$, flanked by poorly resolved ${ }^{195} \mathrm{Pt}$ satellites $\left({ }^{2} J_{\mathrm{CPt}} \mathrm{ca} .12\right.$ $\mathrm{Hz})^{22}$

3. cis $-\left[\left(\mathrm{PPh}_{3}\right)_{2} \mathrm{PtNH}=\mathrm{C}(\mathrm{Me})\{9-\mathrm{MeAd}(-2 \mathrm{H})\}\right] \mathrm{NO}_{3}$ (3b). $\mathrm{A}$ suspension of cis-[( $\left.\left.\mathrm{PPh}_{3}\right)_{2} \mathrm{Pt}(\mu-\mathrm{OH})\right]_{2}\left(\mathrm{NO}_{3}\right)_{2}, \mathbf{1 b},(211 \mathrm{mg}, 0.132$ mmol) and 9-MeAd (40 mg, $0.27 \mathrm{mmol})$ in $\mathrm{CH}_{3} \mathrm{CN}(5 \mathrm{~mL})$ was stirred for $2 \mathrm{~h}$ at ca. $25^{\circ} \mathrm{C}$. Addition of $\mathrm{Et}_{2} \mathrm{O}(20 \mathrm{~mL})$ to the resulting solution afforded a pale yellow solid which was purified by dissolution in hot $\mathrm{CH}_{3} \mathrm{CN}$, filtered to eliminate trace amounts of undissolved material, and precipitated with $\mathrm{Et}_{2} \mathrm{O}$. The pale yellow solid was collected by filtration, washed with $\mathrm{Et}_{2} \mathrm{O}$, and dried under vacuum. The yield of pure 3b was $169 \mathrm{mg}$, yield 65\%. Small crystals, suitable for single-crystal X-ray diffraction, were obtained by vapor diffusion of $\mathrm{Et}_{2} \mathrm{O}$ into a $\mathrm{CH}_{3} \mathrm{CN}$ solution of $\mathbf{3 b}$. Elemental Anal. Calcd for $\mathrm{C}_{44} \mathrm{H}_{39} \mathrm{~N}_{7} \mathrm{O}_{3} \mathrm{P}_{2} \mathrm{Pt}$ : C, 54.43; H, 4.05; N, 10.10 . Found: $\mathrm{C}, 53.42 ; \mathrm{H}, 3.77 ; \mathrm{N}, 10.08 .\left\{{ }^{1} \mathrm{H}\right\}{ }^{31} \mathrm{P}$ NMR in $\mathrm{CD}_{3} \mathrm{CN}$ : $\mathrm{AB}$ multiplet at $\delta 10.27\left({ }^{1} J_{\mathrm{PPt}}=3398 \mathrm{~Hz}\right)$ and $11.47\left({ }^{1} J_{\mathrm{PPt}}=3269\right.$ $\mathrm{Hz}$ ) with ${ }^{2} J_{\mathrm{PP}}=24.5 \mathrm{~Hz}$. Inverse-detected ${ }^{13} \mathrm{C}$ NMR (in DMSO$d_{6}$ ): (9-MeAd resonances) 155.5 (C-2), 148.3 (C-4), 125.2 (C-5), 149.0 (C-6), $144.1(\mathrm{C}-8), 29.4\left(\mathrm{NCH}_{3}\right) . \mathrm{PPh}_{3}$ resonances: 133.2126.8. $\mathrm{CH}_{3} \mathrm{CN}$ resonances: $\delta 163.0$ and 27.0. $\left\{{ }^{1} \mathrm{H}\right\}{ }^{31} \mathrm{P}$ NMR in $\mathrm{CDCl}_{3}$ : $\mathrm{AB}$ multiplet at $\delta 11.33\left({ }^{1} J_{\mathrm{PPt}}=3385 \mathrm{~Hz}\right)$ and $10.86\left({ }^{1} J_{\mathrm{PPt}}\right.$ $=3369 \mathrm{~Hz})$ with ${ }^{2} J_{\mathrm{PP}}=24.7 \mathrm{~Hz} .\left\{{ }^{1} \mathrm{H}\right\}{ }^{31} \mathrm{P}$ NMR in DMSO- $d_{6}$ : $\mathrm{AB}$ multiplet at $\delta 10.91\left({ }^{1} J_{\mathrm{PPt}}=3260 \mathrm{~Hz}\right)$ and $9.47\left({ }^{1} J_{\mathrm{PPt}}=3428\right.$ $\mathrm{Hz})$ with ${ }^{2} J_{\mathrm{PP}}=24.6 \mathrm{~Hz}$.

4. $c i s-\left[\left(\mathrm{PMePh}_{2}\right)_{2} \mathrm{PtNH}=\mathrm{C}(\mathrm{Me})\{1-\mathrm{MeCy}(-\mathbf{2 H})\}\right] \mathrm{NO}_{3}$ (5a). $\mathrm{A}$ suspension of $\mathbf{1 a}(233 \mathrm{mg}, 0.17 \mathrm{mmol})$ and $1-\mathrm{MeCy}(44 \mathrm{mg}, 0.3$ mmol) in $\mathrm{CH}_{3} \mathrm{CN}(7 \mathrm{~mL})$ was stirred at room temperature for 30 min. Addition of $\mathrm{Et}_{2} \mathrm{O}$ to the resulting pale yellow solution afforded a white solid which was separated by filtration and recrystallized from $\mathrm{CH}_{3} \mathrm{CN} / \mathrm{Et}_{2} \mathrm{O}$. The yield of pure 5a was $230 \mathrm{mg}$ (yield $81 \%$ ). Elemental Anal. Calcd for $\mathrm{C}_{33} \mathrm{H}_{35} \mathrm{~N}_{5} \mathrm{O}_{4} \mathrm{P}_{2} \mathrm{Pt}$ : C, 48.18; H, 4.29; N, 
Longato et al.

Table 3. ${ }^{1} \mathrm{H}$ NMR Data ( $\delta$ in ppm, $J$ in $\mathrm{Hz}$ ) for Complexes 3a and $\mathbf{3 b}$ in Various Solvents and Temperatures

\begin{tabular}{|c|c|c|c|c|c|c|c|c|}
\hline compound & $\begin{array}{l}\text { solvent } \\
\left({ }^{\circ} \mathrm{C}\right)\end{array}$ & $\mathrm{H}(2)$ & $\mathrm{H}(8)$ & $\mathrm{NH}$ & $\mathrm{NCH}_{3}$ & $\mathrm{~N}=\mathrm{CCH}_{3}$ & $\mathrm{PMe}$ & $\mathrm{Ph}$ \\
\hline $3 a$ & $\begin{array}{l}\text { DMSO- } d_{6} \\
(25)\end{array}$ & $\begin{array}{l}8.16 \text { app t } \\
\left({ }^{4} J_{\mathrm{HP}}=1.8\right)\end{array}$ & $8.03 \mathrm{~s}$ & 5.84 br s & $3.52 \mathrm{~s}$ & $2.01 \mathrm{~s}$ & $\begin{array}{l}2.23 \mathrm{~d} \\
\left({ }^{2} J_{\mathrm{HP}}=10.0\right) \\
1.91 \mathrm{~d} \\
\left({ }^{2} J_{\mathrm{HP}}=9.5\right)\end{array}$ & $7.64-7.28$ \\
\hline 3a & $\begin{array}{l}\mathrm{CD}_{3} \mathrm{CN} \\
(25)\end{array}$ & $\begin{array}{l}8.05 \text { app t } \\
\left({ }^{4} J_{\mathrm{HP}}=1.7\right. \\
\left.{ }^{3} J_{\mathrm{HPt}}=17.1\right)\end{array}$ & $7.87 \mathrm{~s}$ & 5.39 br s & $3.51 \mathrm{~s}$ & $1.98 \mathrm{~s}$ & $\begin{array}{l}2.01 \mathrm{~d} \\
\left({ }^{2} J_{\mathrm{HP}}=10.2\right) \\
1.81 \mathrm{~d} \\
\left({ }^{2} J_{\mathrm{HP}}=9.6\right)\end{array}$ & $7.66-7.30$ \\
\hline 3a & $\begin{array}{l}\mathrm{CD}_{3} \mathrm{CN} \\
(-40)\end{array}$ & $\begin{array}{l}7.94 \text { app t } \\
\left({ }^{4} J_{\mathrm{HP}}=1.8\right)\end{array}$ & $7.89 \mathrm{~s}$ & $\begin{array}{l}5.36 \text { app dd } \\
\left(J_{\mathrm{HP}}=4-6\right)\end{array}$ & $3.45 \mathrm{~s}$ & $1.92 \mathrm{~s}$ & $\begin{array}{l}1.97 \mathrm{~d} \\
\left({ }^{2} J_{\mathrm{HP}}=8.7\right) \\
1.76 \mathrm{~d} \\
\left({ }^{2} J_{\mathrm{HP}}=9.3\right)\end{array}$ & $7.64-7.22$ \\
\hline 3a & $\begin{array}{l}\mathrm{CDCl}_{3} \\
(25)\end{array}$ & $7.85 \mathrm{~s}$ & $8.12 \mathrm{~s}$ & 5.38 br s & $3.62 \mathrm{~s}$ & $2.07 \mathrm{~s}$ & $\begin{array}{l}2.17 \mathrm{~d} \\
\left({ }^{2} J_{\mathrm{HP}}=9.2\right) \\
1.83 \\
\left({ }^{2} J_{\mathrm{HP}}=8.6\right)\end{array}$ & $7.64-7.28$ \\
\hline $3 b$ & $\begin{array}{l}\text { DMSO- } d_{6} \\
(25)\end{array}$ & $\begin{array}{l}8.08 \text { app t } \\
\left({ }^{4} J_{\mathrm{HP}}=1-2\right)\end{array}$ & $8.17 \mathrm{~s}$ & $\begin{array}{l}6.11 \mathrm{dd} \\
\left({ }^{3} J_{\mathrm{HP}}=3-7\right)\end{array}$ & $3.54 \mathrm{~s}$ & $1.87 \mathrm{~s}$ & & $7.64-7.14$ \\
\hline $3 b$ & $\begin{array}{l}\mathrm{CD}_{3} \mathrm{CN} \\
(25)\end{array}$ & $\begin{array}{l}8.08 \text { app t } \\
\left({ }^{4} J_{\mathrm{HP}}=1.8\right. \\
\left.{ }^{3} J_{\mathrm{HPt}}=18\right)\end{array}$ & $7.86 \mathrm{~s}$ & $5.69 \mathrm{br} \mathrm{s}$ & $3.46 \mathrm{~s}$ & $1.89 \mathrm{~s}$ & & $7.61-7.17$ \\
\hline $3 b$ & $\begin{array}{l}\mathrm{CD}_{3} \mathrm{CN} \\
(-40)\end{array}$ & $\begin{array}{l}7.94 \text { app t } \\
\left({ }^{4} J_{\mathrm{HP}}=1.8\right)\end{array}$ & $7.62 \mathrm{~s}$ & $\begin{array}{l}5.93 \text { app dd } \\
\left({ }^{3} J_{\mathrm{HP}}=5.3\right)\end{array}$ & $3.42 \mathrm{~s}$ & $1.86 \mathrm{~s}$ & & $7.50-7.16$ \\
\hline $3 \mathbf{b}$ & $\begin{array}{l}\mathrm{CDCl}_{3} \\
(25)\end{array}$ & $\begin{array}{l}8.11 \text { app t } \\
\left({ }^{4} J_{\mathrm{HP}}=1.5\right)\end{array}$ & $7.81 \mathrm{~s}$ & $5.44 \mathrm{br} \mathrm{s}$ & $3.50 \mathrm{~s}$ & $1.87 \mathrm{~s}$ & & $7.61-7.17$ \\
\hline
\end{tabular}

Table 4. ${ }^{15} \mathrm{~N}$ NMR Data $(\delta$ in ppm, $J$ in $\mathrm{Hz}$ ) for Complexes 3a,b, 2a, and $\mathbf{4 b}$ in DMSO- $d_{6}$

\begin{tabular}{|c|c|c|c|c|c|c|}
\hline complex & $\mathrm{N}(1)$ & $\mathrm{N}(3)$ & $\mathrm{N}(6)$ & $\mathrm{N}(7)$ & $\mathrm{N}(9)$ & $\mathrm{CH}_{3} \mathrm{CN}$ \\
\hline $3 \mathbf{a}$ & $\begin{array}{l}-198.0 \\
\left({ }^{2} J_{\mathrm{NP}}=39\right)\end{array}$ & -138.6 & -172.1 & -127.1 & -220.8 & $\begin{array}{l}-233.5 \\
\left({ }^{2} J_{\mathrm{NP}}=53\right)\end{array}$ \\
\hline $3 b$ & $\begin{array}{l}-200.0 \\
\left({ }^{2} J_{\mathrm{NP}}=60\right)\end{array}$ & -138.8 & -172.0 & -126.7 & -221.0 & $\begin{array}{l}-234.5 \\
\left({ }^{2} J_{\mathrm{NP}}=58\right)\end{array}$ \\
\hline $2 a$ & -132.0 & -155.3 & $\begin{array}{l}-244.7 \\
\left({ }^{2} J_{\mathrm{NP}}=50\right)\end{array}$ & $\begin{array}{l}-190.4 \\
\left({ }^{2} J_{\mathrm{NP}}=60\right)\end{array}$ & -217.8 & \\
\hline $4 b$ & -131.5 & -153.2 & $\begin{array}{l}-243.1 \\
\left({ }^{2} J_{\mathrm{NP}}=56\right)\end{array}$ & -191.5 & -216.5 & \\
\hline
\end{tabular}

Table 5. Selected Bond Lengths ( $\AA$ ) and Angles (Deg) in the Cation of $5 a$

$\begin{array}{llll}\mathrm{Pt}-\mathrm{N}(3) & 2.097(4) & \mathrm{N}(3)-\mathrm{C}(4) & 1.358(7) \\ \mathrm{Pt}-\mathrm{N}(2) & 2.036(4) & \mathrm{N}(3)-\mathrm{C}(2) & 1.388(6) \\ \mathrm{Pt}-\mathrm{P}(1) & 2.279(1) & \mathrm{N}(4)-\mathrm{C}(3) & 1.337(7) \\ \mathrm{Pt}-\mathrm{P}(2) & 2.262(1) & \mathrm{N}(4)-\mathrm{C}(4) & 1.356(7) \\ \mathrm{N}(2)-\mathrm{C}(3) & 1.295(7) & \mathrm{C}(3)-\mathrm{C}(7) & 1.503(8) \\ \mathrm{N}(2)-\mathrm{Pt}-\mathrm{N}(3) & 83.12(19) & \mathrm{C}(2)-\mathrm{N}(3)-\mathrm{C}(4) & 120.9(4) \\ \mathrm{N}(2)-\mathrm{Pt}-\mathrm{P}(1) & 169.24(14) & \mathrm{C}(2)-\mathrm{N}(3)-\mathrm{Pt} & 117.8(3) \\ \mathrm{N}(2)-\mathrm{Pt}-\mathrm{P}(2) & 88.93(15) & \mathrm{C}(4)-\mathrm{N}(3)-\mathrm{Pt} & 121.3(4) \\ \mathrm{N}(3)-\mathrm{Pt}-\mathrm{P}(1) & 95.60(12) & \mathrm{N}(2)-\mathrm{C}(3)-\mathrm{N}(4) & 126.7(5) \\ \mathrm{N}(3)-\mathrm{Pt}-\mathrm{P}(2) & 172.00(12) & \mathrm{N}(2)-\mathrm{C}(3)-\mathrm{C}(7) & 118.7(5) \\ \mathrm{P}(1)-\mathrm{Pt}-\mathrm{P}(2) & 92.37(5) & \mathrm{N}(4)-\mathrm{C}(3)-\mathrm{C}(7) & 114.6(5) \\ \mathrm{C}(3)-\mathrm{N}(2)-\mathrm{Pt} & 124.9(4) & \mathrm{N}(3)-\mathrm{C}(4)-\mathrm{N}(4) & 125.6(5) \\ \mathrm{C}(3)-\mathrm{N}(4)-\mathrm{C}(4) & 122.6(5) & \mathrm{N}(3)-\mathrm{C}(4)-\mathrm{C}(5) & 117.8(5) \\ & & \mathrm{N}(4)-\mathrm{C}(4)-\mathrm{C}(5) & 116.6(5)\end{array}$

8.51. Found: $\mathrm{C}, 48.01 ; \mathrm{H}, 4.08 ; \mathrm{N}, 8.47 .{ }^{1} \mathrm{H}$ NMR data are collected in Table 6. $\left\{{ }^{1} \mathrm{H}\right\}^{31} \mathrm{P}$ NMR in $\mathrm{CD}_{2} \mathrm{Cl}_{2}$ at $27{ }^{\circ} \mathrm{C}$ : $\mathrm{AB}$ multiplet at $\delta$ $-6.06\left({ }^{1} J_{\mathrm{PPt}}=3325 \mathrm{~Hz}\right)$ and $-9.43\left({ }^{1} J_{\mathrm{PPt}}=3311 \mathrm{~Hz}\right)$ with ${ }^{2} J_{\mathrm{PP}}=$ $27.2 \mathrm{~Hz}$; at $-40{ }^{\circ} \mathrm{C}, \mathrm{AB}$ multiplet at $\delta-4.65\left({ }^{1} J_{\mathrm{PPt}}=3280 \mathrm{~Hz}\right)$ and $-6.00\left({ }^{1} J_{\mathrm{PPt}}=3270 \mathrm{~Hz}\right)$ with ${ }^{2} J_{\mathrm{PP}}=28.1 \mathrm{~Hz}$ and $\mathrm{AX}$ multiplet at $\delta-7.15\left({ }^{1} J_{\mathrm{PPt}}=3330 \mathrm{~Hz}\right)$ and $-13.05\left({ }^{1} J_{\mathrm{PPt}}=3330 \mathrm{~Hz}\right)$ with ${ }^{2} J_{\mathrm{PP}}=26.4 \mathrm{~Hz}$, with relative intensities $1.2: 1$, respectively. $\left\{{ }^{1} \mathrm{H}\right\}^{13} \mathrm{C}$ NMR (in $\mathrm{CD}_{2} \mathrm{Cl}_{2}$ at $27{ }^{\circ} \mathrm{C}$ ): 165.75 (s, $\mathrm{CH}_{3} \mathrm{CN}$ ), 161.45 (s, C-4), 155.21 (s, C-2), 144.37 (s, C-6), $104.22\left(\mathrm{~d},{ }^{4} J_{\mathrm{CP}}=3.5 \mathrm{~Hz}, \mathrm{C}-5\right)$, $37.81\left(\mathrm{~s}, \mathrm{NCH}_{3}\right) ; 133.0-125.5$ (complex multiplets, $\mathrm{PMe} P h_{2}$ ), 12.39 $\left(\mathrm{d},{ }^{1} J_{\mathrm{CP}}=42.7 \mathrm{~Hz}, \mathrm{PMePh}\right) .\left\{{ }^{1} \mathrm{H}\right\}^{31} \mathrm{P} \mathrm{NMR}$ in $\mathrm{CD}_{3} \mathrm{CN}$ at $27{ }^{\circ} \mathrm{C}$ : $\mathrm{AB}$ multiplet at $\delta-5.86\left(\mathrm{~d},{ }^{2} J_{\mathrm{PP}}=27.2 \mathrm{~Hz},{ }^{1} J_{\mathrm{PPt}}=3350 \mathrm{~Hz}\right)$ and $-8.80\left(\mathrm{~s},{ }^{1} J_{\mathrm{PPt}}=3345 \mathrm{~Hz}\right)$; at $-40{ }^{\circ} \mathrm{C}, \mathrm{AB}$ multiplet (relative intensity $58 \%)$ at $\delta-4.34\left({ }^{1} J_{\mathrm{PPt}}=3290 \mathrm{~Hz}\right),-5.33\left({ }^{1} J_{\mathrm{PPt}}=3280\right.$ $\mathrm{Hz}$ ) with ${ }^{2} J_{\mathrm{PP}}=28.4 \mathrm{~Hz}$ and $\mathrm{AX}$ multiplet (relative intensity $42 \%$ ) at $\delta-6.31\left({ }^{1} J_{\mathrm{PPt}}=3360 \mathrm{~Hz}\right),-11.16\left({ }^{1} J_{\mathrm{PPt}}=3360 \mathrm{~Hz}\right)$ with ${ }^{2} J_{\mathrm{PP}}$ $=26.4 \mathrm{~Hz} .\left\{{ }^{1} \mathrm{H}\right\}{ }^{31} \mathrm{P}$ NMR in DMSO- $d_{6}$ at $27{ }^{\circ} \mathrm{C}: \mathrm{AB}$ multiplet at $\delta-5.38\left(\mathrm{~d},{ }^{2} J_{\mathrm{PP}}=26.7 \mathrm{~Hz},{ }^{1} J_{\mathrm{PPt}}=3334 \mathrm{~Hz}\right)$ and $-8.38\left(\mathrm{~s},{ }^{1} J_{\mathrm{PPt}}\right.$ $=3310 \mathrm{~Hz})$. In a second experiment, carried out in $\mathrm{CD}_{3} \mathrm{CN}(0.5$ $\mathrm{mL})$, a suspension of $44 \mathrm{mg}$ of $\mathbf{1 a}$ and $1-\mathrm{MeCy}(8.1 \mathrm{mg})$ was stirred for $1 \mathrm{~h}$, at room temperature. The resulting pale yellow solution, after $24 \mathrm{~h}$, separated pale yellow crystals of $\mathbf{5 a}$ (ca. $30 \mathrm{mg}$ ) which were used for the X-ray analysis.

5. $\operatorname{cis}-\left[\left(\mathrm{PPh}_{3}\right)_{2} \mathbf{P t N H}=\mathrm{C}(\mathrm{Me})\{1-\mathrm{MeCy}(-\mathbf{2 H})\}\right] \mathrm{NO}_{3}$ (5b). $\mathrm{A}$ suspension of $\mathbf{1 b}(81 \mathrm{mg}, 0.05 \mathrm{mmol})$ and 1-MeCy (12.7 mg, 0.10 mmol) in $\mathrm{CH}_{3} \mathrm{CN}$ (4 mL) was stirred at room temperature for 12 h. Addition of $\mathrm{Et}_{2} \mathrm{O}$ to the resulting pale yellow solution afforded a pale yellow solid which was separated by filtration and recrystallized from $\mathrm{CH}_{3} \mathrm{CN} / \mathrm{Et}_{2} \mathrm{O}$. The yield of pure $\mathbf{5 b}$ was $58 \mathrm{mg}$ (yield $61 \%$ ). Elemental Anal. Calcd for $\mathrm{C}_{43} \mathrm{H}_{39} \mathrm{~N}_{5} \mathrm{O}_{4} \mathrm{P}_{2} \mathrm{Pt}$ : C, 54.54; $\mathrm{H}$, 4.16; N, 7.39. Found: C, 54.40; H, 4.10; N, 7.29. $\left\{{ }^{1} \mathrm{H}\right\}^{31} \mathrm{P}$ NMR in $\mathrm{CD}_{3} \mathrm{CN}$ at $27{ }^{\circ} \mathrm{C}$ : $\mathrm{AB}$ multiplet at $\delta 8.81\left({ }^{1} J_{\mathrm{PPt}}=3476 \mathrm{~Hz}\right)$ and $8.01\left({ }^{1} J_{\mathrm{PPt}}=3432 \mathrm{~Hz}\right)$ with ${ }^{2} J_{\mathrm{PP}}=25.0 \mathrm{~Hz}$; in $\mathrm{CDCl}_{3}, \mathrm{AB}$ multiplet at $\delta 8.99\left({ }^{1} J_{\mathrm{PPt}}=3477 \mathrm{~Hz}\right)$ and $7.77\left({ }^{1} J_{\mathrm{PPt}}=3419 \mathrm{~Hz}\right)$ with ${ }^{2} J_{\mathrm{PP}}$ $=24.8 \mathrm{~Hz}$; DMSO- $d_{6}, \mathrm{AB}$ multiplet at $\delta 9.48\left({ }^{1} J_{\mathrm{PPt}}=3444 \mathrm{~Hz}\right)$ and $8.45\left({ }^{1} J_{\mathrm{PPt}}=3442 \mathrm{~Hz}\right)$ with ${ }^{2} J_{\mathrm{PP}}=25.0 \mathrm{~Hz}$.

6. Decomposition of $5 \mathrm{a}$ in Chlorinated Solvents. A solution of $5 \mathbf{a}(139 \mathrm{mg})$ in $\mathrm{CH}_{2} \mathrm{Cl}_{2}(5 \mathrm{~mL})$ was left at room temperature for 2 weeks. Addition of $\mathrm{Et}_{2} \mathrm{O}$ afforded a white precipitate which was recovered by filtration, washed with $\mathrm{Et}_{2} \mathrm{O}$, and dried under vacuum. The elemental analysis of the solid (71 $\mathrm{mg}$ ), after recrystallization from $\mathrm{CH}_{2} \mathrm{Cl}_{2} / \mathrm{Et}_{2} \mathrm{O}$, shows a composition significantly different from the values calculated for the expected cytosine complex $\left[\left(\mathrm{PMePh}_{2}\right)_{2}-\right.$ $\mathrm{Pt}\{1-\mathrm{MeCy}(-\mathrm{H})\}]_{n}\left(\mathrm{NO}_{3}\right)_{n}$. Calcd for $\mathrm{C}_{31} \mathrm{H}_{32} \mathrm{~N}_{4} \mathrm{O}_{4} \mathrm{P}_{2} \mathrm{Pt}$ : C, 47.63; H, 4.13; N, 7.17. Found: C, 45.99; H, 4.13; N, 6.84. ${ }^{1} \mathrm{H}$ NMR in $\mathrm{CDCl}_{3}$ at $27{ }^{\circ} \mathrm{C}(\delta, \mathrm{ppm}):(1-\mathrm{MeCy}(-\mathrm{H})) 6.73\left(\mathrm{~d},{ }^{3} J_{\mathrm{HH}}=5.1 \mathrm{~Hz}\right.$, $1 \mathrm{H}, \mathrm{H}(6)), 6.58(\mathrm{~s}, 1 \mathrm{H}, \mathrm{NH}), 6.34\left(\mathrm{~d},{ }^{3} J_{\mathrm{HH}}=6.0 \mathrm{~Hz}, 1 \mathrm{H}, \mathrm{H}(5)\right)$, 2.89 (s, $\left.3 \mathrm{H}, \mathrm{NCH}_{3}\right) ; \mathrm{PMePh}_{2}, 8.06-7.04$ (cm 20 H, PPh), 2.75 (d, ${ }^{2} J_{\mathrm{HP}}=10 \mathrm{~Hz}, 3 \mathrm{H}, \mathrm{PMe}$ ), 2.28 (br s, $\left.3 \mathrm{H}, \mathrm{PMe}\right) .\left\{{ }^{1} \mathrm{H}\right\}{ }^{31} \mathrm{P}$ NMR in $\mathrm{CDCl}_{3}$ at $27{ }^{\circ} \mathrm{C}$ : apparent doublet at $\delta-11.05\left({ }^{2} J_{\mathrm{PP}}=21 \mathrm{~Hz}\right.$, ${ }^{1} J_{\mathrm{PPt}}=3356 \mathrm{~Hz}$ ) and an extremely broad resonance in the range 
Table 6. ${ }^{1} \mathrm{H}$ NMR Data $(\delta$ in ppm, $J$ in $\mathrm{Hz}$ ) for Complexes $\mathbf{5 a}$ and $\mathbf{5 b}$ in Various Solvents and Temperatures

\begin{tabular}{|c|c|c|c|c|c|c|c|c|}
\hline compound & $\begin{array}{l}\text { solvent } \\
T\left({ }^{\circ} \mathrm{C}\right)\end{array}$ & $\mathrm{H}(5)$ & $\mathrm{H}(6)$ & $\mathrm{NH}$ & $\mathrm{NCH}_{3}$ & $\mathrm{~N}=\mathrm{CCH}_{3}$ & $\mathrm{PMe}$ & $\mathrm{Ph}$ \\
\hline $5 a$ & $\begin{array}{l}\text { DMSO- } d_{6} \\
(25)\end{array}$ & $\begin{array}{l}5.84 \mathrm{dd} \\
\left({ }^{3} J_{\mathrm{HH}}=7.1 ;\right. \\
\left.{ }^{5} J_{\mathrm{HP}}=1.3\right)\end{array}$ & $\begin{array}{l}7.27 \mathrm{~d} \\
\left({ }^{3} J_{\mathrm{HH}}=7.1\right)\end{array}$ & 6.29 br s & $3.62 \mathrm{~s}$ & $1.95 \mathrm{~s}$ & $\begin{array}{l}2.13 \mathrm{~d} \\
\left({ }^{2} J_{\mathrm{HP}}=10.8\right)\end{array}$ & $7.64-7.28$ \\
\hline $5 a$ & $\begin{array}{l}\mathrm{CD}_{3} \mathrm{CN} \\
(25)\end{array}$ & $\begin{array}{l}5.81 \mathrm{dd} \\
\left({ }^{3} J_{\mathrm{HH}}=7.1 ;\right. \\
\left.{ }^{5} J_{\mathrm{HP}}=1.4\right)\end{array}$ & $\begin{array}{l}6.93 \mathrm{~d} \\
\left({ }^{3} J_{\mathrm{HH}}=7.1\right)\end{array}$ & $5.50 \mathrm{br} \mathrm{s}$ & $2.56 \mathrm{~s}$ & $1.89 \mathrm{~s}$ & $\begin{array}{l}\text { ca. } 2 \text { br s } \\
1.89 \mathrm{~d} \\
\left({ }^{2} J_{\mathrm{HP}}=10.5\right) \\
1.93 \mathrm{~d} \\
\left({ }^{2} J_{\mathrm{HP}}=10.0\right)\end{array}$ & $7.60-7.29$ \\
\hline $5 \mathbf{a}$ & $\begin{array}{l}\mathrm{CD}_{2} \mathrm{Cl}_{2} \\
(25)\end{array}$ & $\begin{array}{l}5.91 \mathrm{dd} \\
\left({ }^{3} J_{\mathrm{HH}}=7.2 ;\right. \\
\left.{ }^{5} J_{\mathrm{HP}}=1.5\right)\end{array}$ & $\begin{array}{l}7.92 \mathrm{~d} \\
\left({ }^{3} J_{\mathrm{HH}}=7.2\right)\end{array}$ & $5.53 \mathrm{br} \mathrm{s}$ & $2.63 \mathrm{~s}$ & $2.07 \mathrm{~s}$ & $\begin{array}{l}1.84 \mathrm{~d} \\
\left({ }^{2} J_{\mathrm{HP}}=10.0\right) \\
1.93 \mathrm{~d} \\
\left({ }^{2} J_{\mathrm{HP}}=11.0\right)\end{array}$ & $7.62-7.34$ \\
\hline $5 b$ & $\begin{array}{l}\text { DMSO- } d_{6} \\
(25)\end{array}$ & $\begin{array}{l}5.86 \mathrm{dd} \\
\left({ }^{3} J_{\mathrm{HH}}=7\right. \\
\left.{ }^{5} J_{\mathrm{HP}}=0.9\right)\end{array}$ & $\begin{array}{l}7.26 \mathrm{~d} \\
\left({ }^{3} J_{\mathrm{HH}}=7.3\right)\end{array}$ & $\begin{array}{l}7.03 \text { app t } \\
\left({ }^{3} J_{\mathrm{HP}}=4-6\right)\end{array}$ & $3.50 \mathrm{~s}$ & $1.85 \mathrm{~s}$ & & $7.67-7.28$ \\
\hline $5 b$ & $\begin{array}{l}\mathrm{CD}_{3} \mathrm{CN} \\
(25)\end{array}$ & $\begin{array}{l}5.83 \mathrm{dd} \\
\left({ }^{3} J_{\mathrm{HH}}=7.2 ;\right. \\
\left.{ }^{5} J_{\mathrm{HP}}=1.2\right)\end{array}$ & $\begin{array}{l}6.91 \mathrm{~d} \\
\left({ }^{3} J_{\mathrm{HH}}=7.2\right)\end{array}$ & 5.84 br s & $2.49 \mathrm{~s}$ & $1.95 \mathrm{~s}$ & & $7.80-7.23$ \\
\hline $5 b$ & $\begin{array}{l}\mathrm{CDCl}_{3} \\
(25)\end{array}$ & $\begin{array}{l}6.02 \mathrm{dd} \\
\left({ }^{3} J_{\mathrm{HH}}=7.0 ;\right. \\
\left.{ }^{5} J_{\mathrm{HP}}=1.5\right)\end{array}$ & $\begin{array}{l}7.20 \mathrm{~d} \\
\left({ }^{3} J_{\mathrm{HH}}=7.1\right)\end{array}$ & 5.72 br s & $2.71 \mathrm{~s}$ & $1.92 \mathrm{~s}$ & & $7.69-7.27$ \\
\hline
\end{tabular}

of -5 to $-10 \mathrm{ppm}$. Similar data were obtained in $\mathrm{CD}_{3} \mathrm{CN}$, even after several days at $45^{\circ} \mathrm{C}$, indicating that the isolated solid does not react with the solvent.

7. Reaction of $c i s-\left[\mathrm{L}_{2} \mathrm{Pt}(\mu-\mathrm{OH})\right]_{2}{ }^{2+}\left(\mathrm{L}=\mathrm{PMe}_{3}, \mathrm{PMe}_{2} \mathrm{Ph}\right)$ with 9-MeAd and 1-MeCy in Acetonitrile. A suspension of cis$\left[\left(\mathrm{PMe}_{3}\right) 2 \mathrm{Pt}(\mu-\mathrm{OH})\right]_{2}\left(\mathrm{ClO}_{4}\right)_{2},(46 \mathrm{mg}, 0.05 \mathrm{mmol})$ and $9-\mathrm{MeAd}(15$ $\mathrm{mg}, 0.1 \mathrm{mmol})$ in $1 \mathrm{~mL}$ of $\mathrm{CD}_{3} \mathrm{CN}$ was stirred at room temperature for $4 \mathrm{~h}$ obtaining a colorless solution. The ${ }^{31} \mathrm{P}$ NMR of the reaction mixture showed the presence of two AB multiplets, flanked by ${ }^{195}$ Pt satellites, at $\delta-27.34\left({ }^{1} J_{\mathrm{PPt}}=3260 \mathrm{~Hz}\right)$ and $-28.56\left({ }^{1} J_{\mathrm{PPt}}=\right.$ $\left.3117 \mathrm{~Hz},{ }^{2} J_{\mathrm{PP}}=25.1 \mathrm{~Hz}\right) \mathrm{ppm}$ and $\delta-29.66\left({ }^{1} J_{\mathrm{PPt}}=3021 \mathrm{~Hz}\right)$ and $-30.86\left({ }^{1} J_{\mathrm{PPt}}=3218 \mathrm{~Hz},{ }^{2} J_{\mathrm{PP}}=26.1 \mathrm{~Hz}\right) \mathrm{ppm}$, with relative intensities ca. 5:1, respectively. In 2 weeks at room temperature the first multiplet quantitatively converted in the second one, attributable to the dinuclear species cis- $\left[\left(\mathrm{PMe}_{3}\right)_{2} \mathrm{Pt}\{9-\mathrm{MeAd}(-\mathrm{H})\}\right]_{2^{-}}$ $\left(\mathrm{ClO}_{4}\right)_{2}$. The solution was then heated at $50{ }^{\circ} \mathrm{C}$ for 5 days. Further changes on the ${ }^{31} \mathrm{P}$ NMR spectrum were not observed. Addition of $\mathrm{Et}_{2} \mathrm{O}$ afforded a white precipitate which was recovered by filtration and dried under vacuum to give $25 \mathrm{mg}$ of solid which was further characterized by elemental analysis, mass spectrometry, and NMR spectroscopy. Anal. Calcd for $\mathrm{C}_{12} \mathrm{H}_{24} \mathrm{~N}_{5} \mathrm{ClO}_{4} \mathrm{P}_{2} \mathrm{Pt}$ : C, 24.23; H, 4.07; N, 11.77. Found: C, 24.17; H, 4.01; N, 11.69. ESI mass spectrum in $\mathrm{CH}_{3} \mathrm{CN}: \mathrm{m} / z, 1089$ due to the monovalent cation [( $\left.\mathrm{PMe}\right)_{2} \mathrm{Pt}\{9-$ $\operatorname{MeAd}(-\mathrm{H})\}]_{2}\left(\mathrm{ClO}_{4}\right)^{+} .{ }^{1} \mathrm{H}$ and $\left\{{ }^{1} \mathrm{H}\right\}{ }^{31} \mathrm{P}$ NMR data (in DMSO- $\left.d_{6}\right)$ of the isolated complex were in agreement those reported for cis$\left[\left(\mathrm{PMe}_{3}\right)_{2} \mathrm{Pt}\{9-\mathrm{MeAd}(-\mathrm{H})\}\right]_{2}\left(\mathrm{NO}_{3}\right)_{2} .{ }^{7}$ With similar procedures, complexes cis- $\left[\left(\mathrm{PMe}_{2} \mathrm{Ph}\right)_{2} \mathrm{Pt}\{9-\mathrm{MeAd}(-\mathrm{H})\}\right]_{3}\left(\mathrm{NO}_{3}\right)_{3}{ }^{8}$ and $c i s-\left[\left(\mathrm{PMe}_{2}-\right.\right.$ $\left.\mathrm{Ph})_{2} \mathrm{Pt}\{1-\mathrm{MeCy}(-\mathrm{H})\}\right]_{3}\left(\mathrm{NO}_{3}\right)_{3}{ }^{13}$ were prepared reacting cis$\left[\left(\mathrm{PMe}_{2} \mathrm{Ph}\right) \mathrm{Pt}(\mu-\mathrm{OH})\right]_{2}\left(\mathrm{NO}_{3}\right)_{2}$ with 9-MeAd and 1-MeCy, respectively, in $\mathrm{CH}_{3} \mathrm{CN}$. No reaction with the solvent was observed, even at 50 ${ }^{\circ} \mathrm{C}$ within 1 week.

X-ray Structure Determinations. Diffraction data were collected on a Stoe \& Cie diffractometer equipped with a STADI4 CCD detector (compounds 3a,b) and on a Nonius DIP-1030H system (5a) graphite-monochromatized Mo K $\alpha$ radiation. Cell refinement, indexing, and scaling of the data sets were carried out using the program $\mathrm{X}-\mathrm{RED}^{14}$ (compounds $\mathbf{3 a}, \mathbf{b}$ ) and by programs Denzo and Scalepack for $\mathbf{5 a} .{ }^{15}$ The structures were solved by direct methods (SHELX86 and SHELXTL NT) and Fourier analyses and

(13) Longato, B.; Montagner, D.; Zangrando, E. Manuscript in preparation. (14) X-RED, version 1.22; Stoe \& Cie: Darmstadt, Germany, 2001. were refined by the full-matrix least-squares method based on $F^{2}$ with all observed reflections. ${ }^{16}$ In $\mathbf{3 b}$ the nitrate oxygen atoms were found disordered over two positions with occupancy factors refined at $0.43(2)$ and $0.57(2)$. The final cycles with fixed contribution of hydrogen atoms at calculated positions converged to final R1 and wR2 factors reported in Table 1.

All the calculations were performed using the WinGX System, version 1.64.05. ${ }^{17}$

\section{Results and Discussion}

Synthesis and Characterization of cis- $\left[\mathrm{L}_{2} \mathrm{PtNH}=\mathrm{C}\right.$ (Me) $\{$ 9-MeAd(-2H) $\}] \mathrm{NO}_{3}\left(\mathrm{~L}=\mathbf{P M e P h}_{2}, \mathbf{3 a} ; \mathbf{P P h}_{3}, \mathbf{3 b}\right)$. We have recently shown that the hydroxo complex cis$\left.\left[\left(\mathrm{PMePh}_{2}\right)_{2} \mathrm{Pt}(\mu-\mathrm{OH})\right)\right]_{2}\left(\mathrm{NO}_{3}\right)_{2}, \mathbf{1 a}$, reacts with the 9-substituted methyladenine (9-MeAd) to give the cyclic trimer $\mathrm{cis}$ $\left[\left(\mathrm{PMePh}_{2}\right)_{2} \mathrm{Pt}\{9-\mathrm{MeAd}(-\mathrm{H})\}\right]_{3}\left(\mathrm{NO}_{3}\right)_{3}, \mathbf{2 a}$, containing the $\mathrm{NH}_{2}$-deprotonated adenine bridging the metal centers through the $\mathrm{N}(1)$ and $\mathrm{N}(6)$ atoms. ${ }^{5}$ When the reaction is carried out in $\mathrm{CH}_{3} \mathrm{CN}$, the initially formed complex $\mathbf{2 a}$, slowly converts into the mononuclear species cis- $\left[\left(\mathrm{PMePh}_{2}\right)_{2} \mathrm{PtNH}=\mathrm{C}(\mathrm{Me})\right.$ $\{9-\mathrm{MeAd}(-2 \mathrm{H})\}] \mathrm{NO}_{3}, \mathbf{3} \mathbf{a}$, according to the reaction shown in Scheme 1.

In the same experimental conditions, complex $\mathbf{1 b}$ forms immediately the insertion product $c i s-\left[\left(\mathrm{PPh}_{3}\right)_{2} \mathrm{PtNH}=\mathrm{C}(\mathrm{Me})\right.$ $\{9-\mathrm{MeAd}(-2 \mathrm{H})\}] \mathrm{NO}_{3}, \mathbf{3 b}$. Both the complexes have been isolated in fairly good yield. X-ray analyses of $\mathbf{3 a}, \mathbf{b}$ show that the insertion of a $\mathrm{CH}_{3} \mathrm{CN}$ molecule into the adenine $\mathrm{Pt}-$ $\mathrm{N}(6)$ bond had occurred, with formation of a six atoms metallocycle, as depicted in Figures 1 and 2, respectively.

A selection of bond distances and angles for these structures is collected in Table 2, indicating a close similarity

(15) Otwinowski, Z.; Minor, W. Processing of X-ray Diffraction Data Collected in Oscillation Mode. In Methods in Enzymology; Carter, C. W., Jr., Sweet, R. M., Eds; Macromolecular Crystallography, Part A; Academic Press: New York, 1997; Vol. 276, pp 307-326.

(16) (a) Sheldrick, G. M. SHELXTL, NT, version 5.10; Brucker Analytical X-ray System: Madison WI, 1999. (b) Sheldrick, G. M. SHELX97 Programs for Crystal Structure Analysis, release 97-2; University of Göttingen: Göttingen, Germany, 1998.

(17) Farrugia, L. J. J. Appl. Crystallogr. 1999, 32, 837-838. 
Longato et al.

Scheme 1

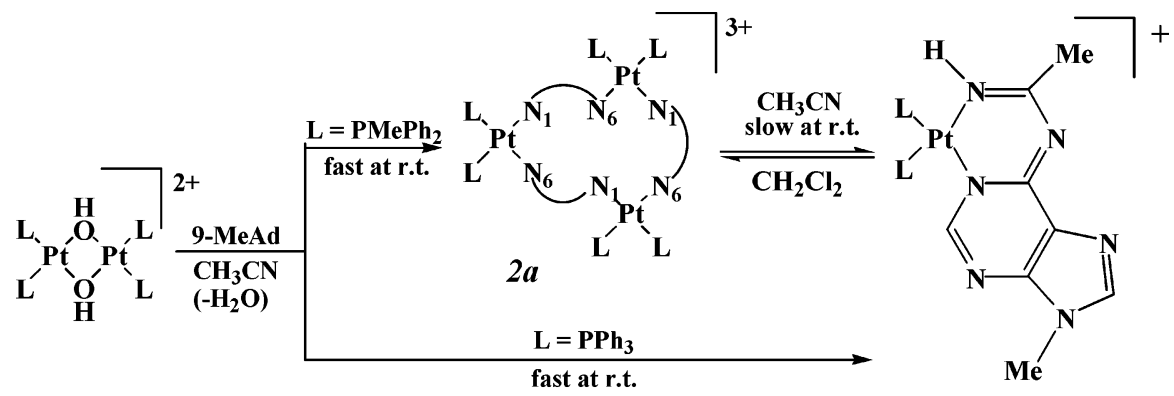

$\mathrm{L}=\mathrm{PMePh}_{2}, 1 a ; \mathrm{PPh}_{3}, 1 b$

of the metal coordination sphere in the two adducts. The platinum is bound to the nucleobase at the $\mathrm{N}(1)$ site, to the inserted acetonitrile nitrogen $\mathrm{N}(2)$, and completes the square planar coordination through phosphorus donors. The $\mathrm{Pt}-$ $\mathrm{N}(1)$ bond lengths are 2.115(5), 2.124(7) $\AA$, in 3a and 3b, respectively, while $\mathrm{Pt}-\mathrm{N}(2)$, of 2.017(5) and 2.011(7) $\AA$, are slightly shorter. The $\mathrm{Pt}-\mathrm{P}$ bond distances average to 2.287(2) $\AA$, a value comparable to that found in the parent complex 2a (2.280(4) $\AA$ )..$^{5}$ The coordination $\mathrm{N}_{2} \mathrm{P}_{2}$ donors show a slight tetrahedral distortion in $\mathbf{3 a}$, with deviations of ca. $\pm 0.10 \AA$ from its mean plane, while they are coplanar in 3b, with the Pt ion slightly displaced by $0.04 \AA$ in both complexes. Figure 3 displays a side view along the $\mathrm{P}(1)-$ $\mathrm{P}(2)$ vector in 3a (a similar conformation is also exhibited by $\mathbf{3 b}$ ), showing the bending orientation assumed by the adenine-acetonitrile moiety with respect to the coordination plane. The dihedral angle formed by the mean planes through these atoms is of $29.2(2)^{\circ}$ and $34.7(2)^{\circ}$, in $\mathbf{3 a}$ and $\mathbf{3 b}$, respectively. The metal displacement from the plane defined by $\mathrm{N}(1) / \mathrm{C}(6) / \mathrm{C}(3) / \mathrm{N}(2)$ is of 0.52 and $0.65 \AA$ in the two complexes.

In all the structures reported the $\mathrm{H}$ atom at acetonitrile nitrogen $\mathrm{N}(2)$ was included considering the evidences achieved from ${ }^{1} \mathrm{H},{ }^{15} \mathrm{~N}$ NMR experiments, since the electron density maps did not allow us to definitely locate this hydrogen atom. The values of bond distance inside the chelating unit (Table 2) indicate a significant $\pi$ electron delocalization, similar to that found in a related platinum(II) metallacycle. ${ }^{18}$ In particular, the $\mathrm{C}(3)-\mathrm{N}(2)$ bond distances for the inserted $\mathrm{CH}_{3} \mathrm{CN}$ molecule present a value typical for a double bond, ${ }^{19}$ being of 1.292(7) and 1.279(11) $\AA$ in $\mathbf{3 a}$ and $\mathbf{3 b}$, respectively. In the $\mathrm{C}(3)-\mathrm{N}(6)-\mathrm{C}(6)-$ $\mathrm{N}(1)-\mathrm{C}(2)$ fragment the distances are slightly longer varying in a range from $1.335(8)$ to $1.397(10) \AA$. All other bond lengths and angles inside the nucleobase are in the usual range. ${ }^{20}$ Compound $\mathbf{3 b}$ shows an intramolecular phenyl stacking occurring between rings of ipso carbon $\mathrm{C}(1 \mathrm{c})$ and $\mathrm{C}(1 \mathrm{~d})$.

(18) Guedes da Silva, M.; Ferreira, C. M. P.; Branco, E. M. P. R. P; Fraústo da Silva, J. J. R.; Pombeiro, A. J. L.; Michelin, R.; Belluco, U.; Bertani, R.; Mozzon, M.; Bombieri, G.; Benetollo, F.; Kukushkin, V. Y. Inorg. Chim. Acta 1997, 265, 267-270.

(19) Allen, F. H.; Kennard, O.; Watson, D. G.; Brammer, L.; Orpen, A. G. J. Chem. Soc., Perkin Trans. 2 1987, S1.

(20) Velders, A. V.; van der Geest, B.; KooiJman, H.; Spek, A.; Haasnoot, J. G.; Reedijk, J. Eur. J. Inorg. Chem. 2001, 369-372.

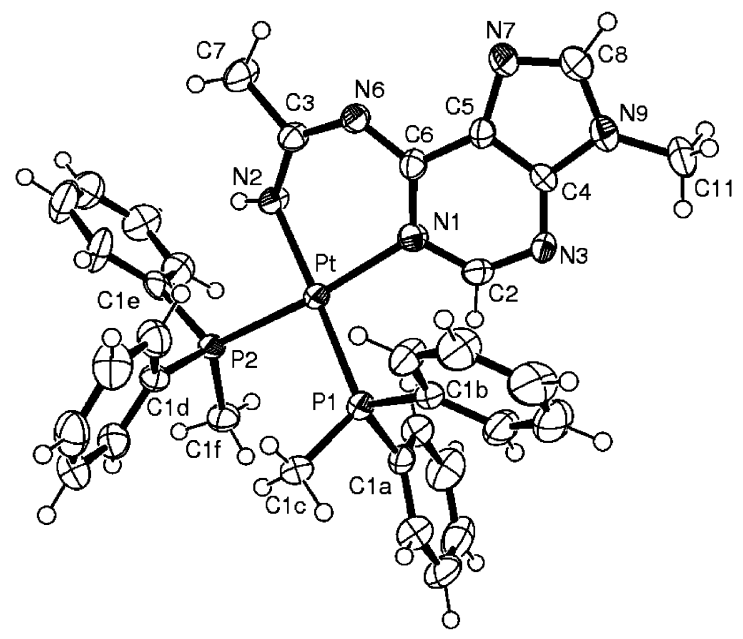

Figure 1. ORTEP drawing (ellipsoid $40 \%$ probability level) of the cation of $3 \mathbf{a}$.

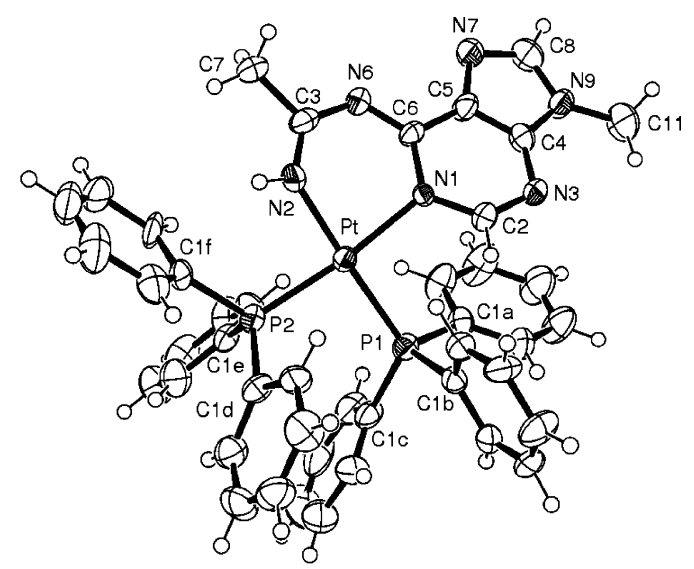

Figure 2. ORTEP drawing (ellipsoid $40 \%$ probability level) of the cation of $\mathbf{3 b}$.

NMR Studies in Solution. The reaction with acetonitrile has been followed by NMR spectroscopy. Figure 4 shows the changes of the ${ }^{31} \mathrm{P}$ NMR spectra of a $\mathrm{CD}_{3} \mathrm{CN}$ solution of $2 \mathbf{a}$ in the course of the transformation to $\mathbf{3 a}$.

A freshly prepared solution of $\mathbf{2 a}$ (trace a) exhibits a sharp $\mathrm{AB}$ multiplet, flanked by the ${ }^{195} \mathrm{Pt}$ satellites, whose parameters are similar to those obtained in chlorinated solvents in which 2a maintains the trinuclear structure found in the solid state. ${ }^{5}$ In several days at room temperature, the multiplet is replaced by a new $\mathrm{AB}$ multiplet at lower field, attributable 

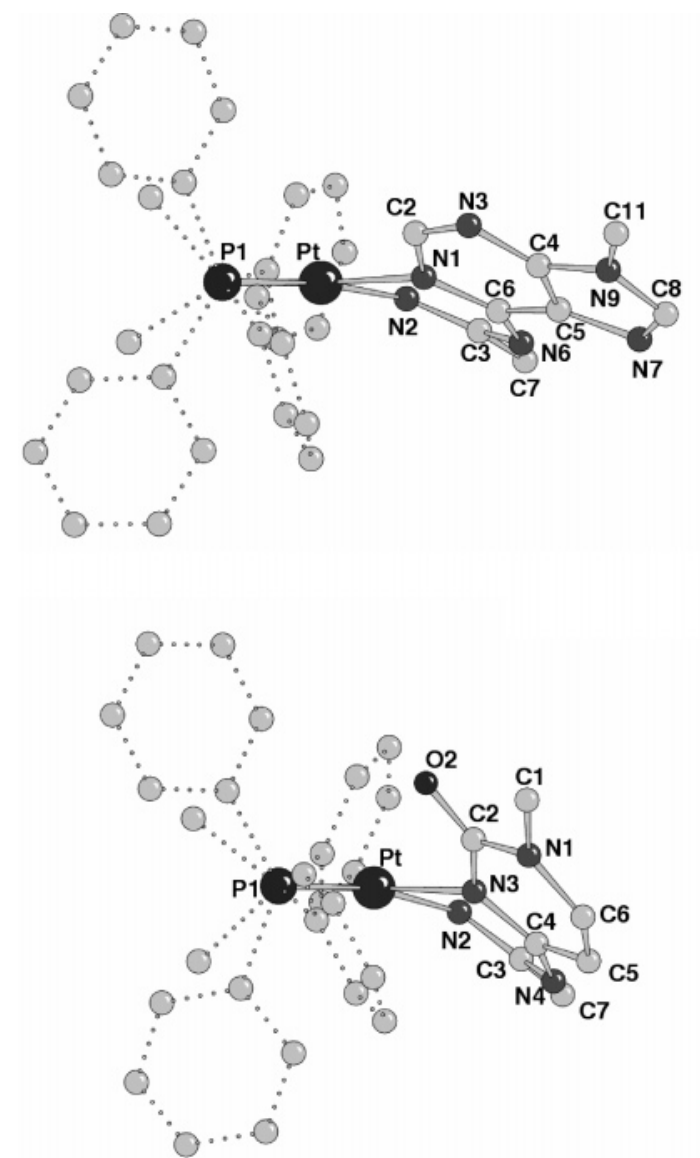

Figure 3. Perspective view of the complexes 3a and 5a along the P1-P2 direction showing the orientation of the nucleobase moiety with respect to the coordination plane.

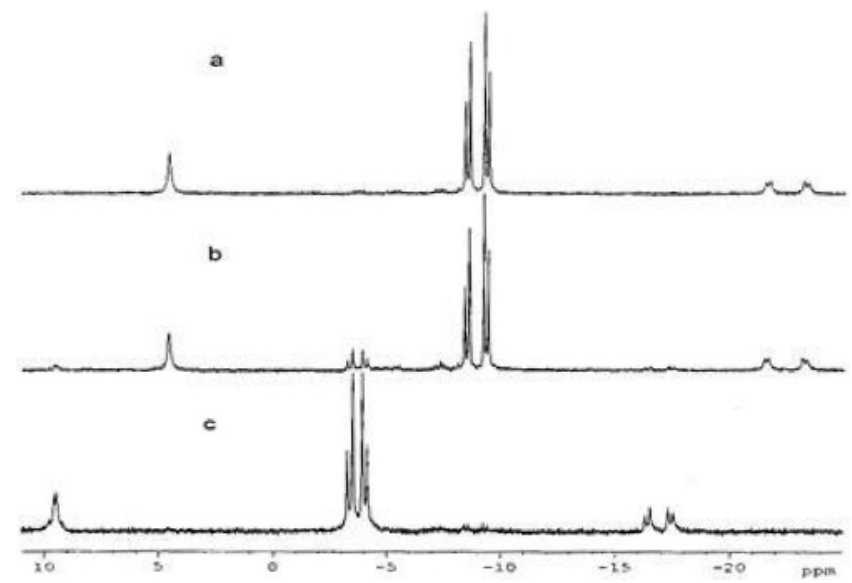

Figure 4. ${ }^{31} \mathrm{P}\left\{{ }^{1} \mathrm{H}\right\}$ NMR spectra of $\mathbf{2 a}$ in $\mathrm{CD}_{3} \mathrm{CN}$ at $27{ }^{\circ} \mathrm{C}$ : (a) fresh solution; (b) after $24 \mathrm{~h}$; (c) after 2 weeks at room temperature.

to complex 3a. The reaction appears complete in 3 weeks (trace c), and no intermediates are detectable during the transformation (trace $b$ ).

The formation of $\mathbf{3 a}$ causes a downfield shift of both the ${ }^{31} \mathrm{P}$ nuclei (ca. $5.2 \mathrm{ppm}$ ), whereas only one of the ${ }^{1} J_{\mathrm{PPt}}$ values changes significantly (from $3372 \mathrm{~Hz}$ in $\mathbf{2 a}$ to $3265 \mathrm{~Hz}$ in 3a). The resonance at $-3.36 \mathrm{ppm}$, whose value of ${ }^{1} J_{\mathrm{PPt}}(3172$ $\mathrm{Hz}$ ) is very similar to one of those found in the parent complex 2a $(3192 \mathrm{~Hz})$, can be attributed to the phosphine in trans to the adenine $\mathrm{N}(1)$ atom. The small change of ${ }^{1} J_{\mathrm{PPt}}$ for this phosphine reflects the invariance of the $\mathrm{Pt}-\mathrm{N}(1)$ bond
Scheme 2

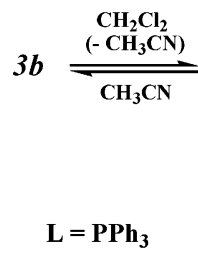

$\mathbf{L}=\mathbf{P P h}_{3}$

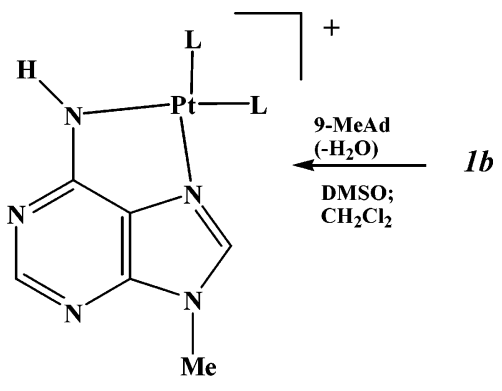

$4 b$ length found in the two complexes $(\mathrm{Pt}-\mathrm{N}(1)=2.117(10)$ $\AA$ (average) $)^{5}$ in 2a, and 2.115(5) $\AA$ in 3a).

In the spectrum of $\mathbf{3 a}$, the methyl protons of the phosphines exhibit distinct and well-resolved resonances, in agreement with the chemical inequivalence of the two ligands. The ${ }^{1} \mathrm{H}$ NMR data, in various solvents and temperatures, are collected in Table 3.

For thorough ${ }^{1} \mathrm{H}$ and ${ }^{13} \mathrm{C}$ resonance assignments the routine 1- and 2-D NMR techniques have been employed. Discrimination between the adenine $\mathrm{H}(2)$ and $\mathrm{H}(8)$ protons was achieved by heteronuclear multiple bond correlations (HMBC) $\mathrm{C} / \mathrm{H}$ experiments. Coordination of the adenine at the $\mathrm{N}(1)$ position is confirmed by the splitting of the resonances $\mathrm{C}(2)$ $\left(\delta 156.3,{ }^{3} J_{\mathrm{CP}}=8.5 \mathrm{~Hz}\right)$ and $\mathrm{H}(2)\left(\delta 8.16,{ }^{4} J_{\mathrm{HP}}=1.8 \mathrm{~Hz}\right)$, due to the coupling with ${ }^{31} \mathrm{P}$ nuclei, in the $\left\{{ }^{1} \mathrm{H}\right\}{ }^{13} \mathrm{C}$ and ${ }^{1} \mathrm{H}$ spectra of 3a. Moreover, $\mathrm{HMBC}{ }^{15} \mathrm{~N},{ }^{1} \mathrm{H}$ experiments show that the resonances at $\delta 5.84$ in $\mathbf{3 a}$ and $6.11 \mathrm{ppm}$ in $\mathbf{3 b}$ correlate with the ${ }^{15} \mathrm{~N}$ resonances at $\delta-233.5$ (3a) and $-234.5 \mathrm{ppm}(\mathbf{3 b})$, indicating that the nitrogen atom of the inserted $\mathrm{CH}_{3} \mathrm{CN}$ is protonated. The pertinent ${ }^{15} \mathrm{~N}$ NMR data are collected in Tables 4, while the spectra are available as Supporting Information.

These attributions are in line with the observation that the $\mathrm{NH}$ resonance at $\delta 6.11$ in $\mathbf{3 b}$ occurs as a doublet of doublets, due to coupling with ${ }^{31} \mathrm{P}$ nuclei, separated by $3-7 \mathrm{~Hz}$, typical values for three-bonds ${ }^{1} \mathrm{H}-{ }^{31} \mathrm{P}$ interactions. Similarly, in $\mathbf{3 a}$ the $\mathrm{NH}$ resonance is a broad singlet at ambient temperature but exhibits fine structure at $-40{ }^{\circ} \mathrm{C}$ (See Table 3). The process responsible of these changes with the temperature was not investigated in detail. However, the possible exchanges of the $\mathrm{NH}$ proton between the $\mathrm{N}(2)$ and the $\mathrm{N}(6)$ atoms for 3a in DMSO- $d_{6}$ was ruled out through a ROESY experiment. The whole of these data supports the conclusion that the anionic ligand in $\mathbf{3 a}$ and $\mathbf{3 b}$, abbreviated as $\mathrm{NH}=$ $\mathrm{C}(\mathrm{Me})\{9-\operatorname{MeAd}(-2 \mathrm{H})\}$, is the deprotonated form of the amidine $N$-(9-methyl-1,9-dihydro-purin-6-ylidene)-acetamidine (see Chart 2).

In solution complexes $\mathbf{3 a}, \mathbf{b}$ are stable only in acetonitrile, in which the inserted $\mathrm{CH}_{3} \mathrm{CN}$ molecule exchanges with the solvent (see experimental). In chlorinated solvents at room temperature, 3a slowly releases the inserted $\mathrm{CH}_{3} \mathrm{CN}$ molecule reforming the trinuclear species $\mathbf{2 a}$ (Scheme 1). The reaction leads to an equilibrium mixture in ca. 4 weeks and, in a solution ca. $3.0 \times 10^{-2} \mathrm{M}\left(\right.$ in $\left.\mathrm{CDCl}_{3}\right)$ the relative intensities of the ${ }^{31} \mathrm{P}$ NMR signals of $\mathbf{2 a}$ and $\mathbf{3 a}$ are ca. $3: 1$. On the 
Longato et al.

Scheme 3

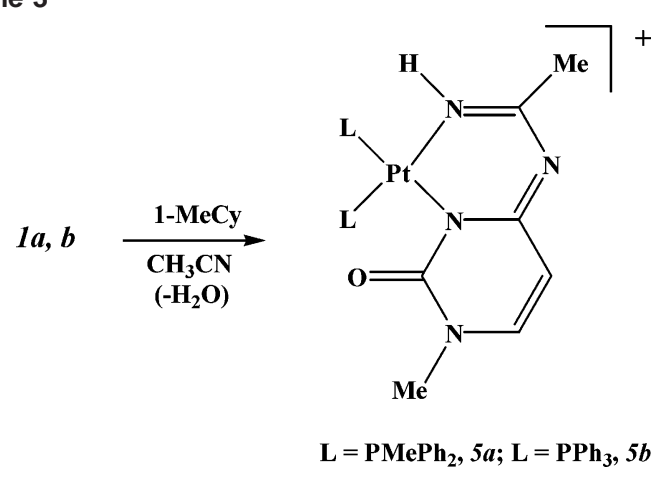

contrary, $\mathbf{3 b}$ in $\mathrm{CH}_{2} \mathrm{Cl}_{2}$ (and DMSO) undergoes a complete decomposition with formation of the mononuclear species 4b (Scheme 2) in which the $\mathrm{NH}_{2}$-deproptonated nucleobase chelates the metal center at the N(6), N(7) sites. ${ }^{21}$

The $\mathrm{PMePh}_{2}$ analogue of this complex was previously characterized as the main component the mixture of products obtained when $\mathbf{2 a}$ is dissolved in DMSO. ${ }^{5}$ Complex $\mathbf{4 b}$ can be isolated as pure product by reacting $\mathbf{1 b}$ with $9-\mathrm{MeAd}$ in dichloromethane. ${ }^{21}$ In $\mathrm{CH}_{3} \mathrm{CN}$ solution $\mathbf{4 b}$ slowly regenerates $\mathbf{3 b}$, indicating a complete reversibility in the insertion reaction of acetonitrile.

Synthesis and Characterization of $c i s-\left[\mathrm{L}_{2} \mathrm{PtNH}=\mathrm{C}\right.$ (Me) $\{1-\mathbf{M e C y}(-2 \mathrm{H})\}] \mathrm{NO}_{3}\left(\mathbf{L}=\mathbf{P M e P h}_{2}, \mathbf{5 a} ; \mathbf{L}=\mathbf{P P h}_{3}\right.$, 5b). A similar metal-promoted coupling of $\mathrm{CH}_{3} \mathrm{CN}$ with the exocyclic nitrogen of 1-methylcytosine (1-MeCy) is observed when complexes 1a,b react with the nucleobase in acetonitrile (Scheme 3).

Mixtures of $\mathbf{1 a}$ or $\mathbf{1 b}$ and $1-\mathrm{MeCy}$ (molar ratio 1:2) in $\mathrm{CH}_{3} \mathrm{CN}$, in a few hours at room temperature, form pale yellow solutions from which the compounds cis $-\left[\mathrm{L}_{2} \mathrm{PtNH}=\right.$ $\mathrm{C}(\mathrm{Me})\{1-\mathrm{MeCy}(-2 \mathrm{H})\}] \mathrm{NO}_{3}\left(\mathrm{~L}=\mathrm{PMePh}_{2}, \mathbf{5 a} ; \mathrm{L}=\mathrm{PPh}_{3}\right.$, 5b) can be separated by crystallization (5a) or precipitation with $\mathrm{Et}_{2} \mathrm{O}(\mathbf{5 b})$. The NMR analysis of the reaction mixture, performed before the separation of the solid, showed the quantitative formation of $\mathbf{5 b}$, whereas for $\mathbf{5 a}$ the yield was ca. 90\%. The remaining product is a species, not yet completely characterized, that can be isolated when $\mathbf{5 a}$ is dissolved in chlorinated solvents (see the Experimental Section).

The X-ray structure analysis of complex 5a (Figure 5) shows the platinum bound to the phosphines and to the nitrogen donors of the adduct obtained from cytosine with a $\mathrm{MeCN}$ solvent molecule, the cytosine coordination site being $\mathrm{N}(3)$.

Both the $\mathrm{Pt}-\mathrm{P}$ and $\mathrm{Pt}-\mathrm{N}$ bond lengths are comparable to those measured in $\mathbf{3 a}$ and $\mathbf{3 b}$ within $2 \sigma$ (Table 5). The $\mathrm{P}_{2} \mathrm{~N}_{2}$ square planar geometry exhibits a slight tetrahedral distortion (atom displacement of about $\pm 0.10 \AA$ ) with the $\mathrm{P}_{2} \mathrm{Pt}$ and $\mathrm{N}_{2} \mathrm{Pt}$ planes forming a dihedral angle of $10.7^{\circ}$. But more severe deformations are detected in the six-membered ring with $\mathrm{Pt}$ and $\mathrm{N}(4)$ located above the mean plane passing through $\mathrm{N}(2), \mathrm{C}(3), \mathrm{C}(4)$, and $\mathrm{N}(3)$ atoms by 0.78 and 0.13 $\AA$, respectively. The latter plane forms an angle of $15.7^{\circ}$ with

(21) Montagner, D.; Longato, B. Unpublished results.

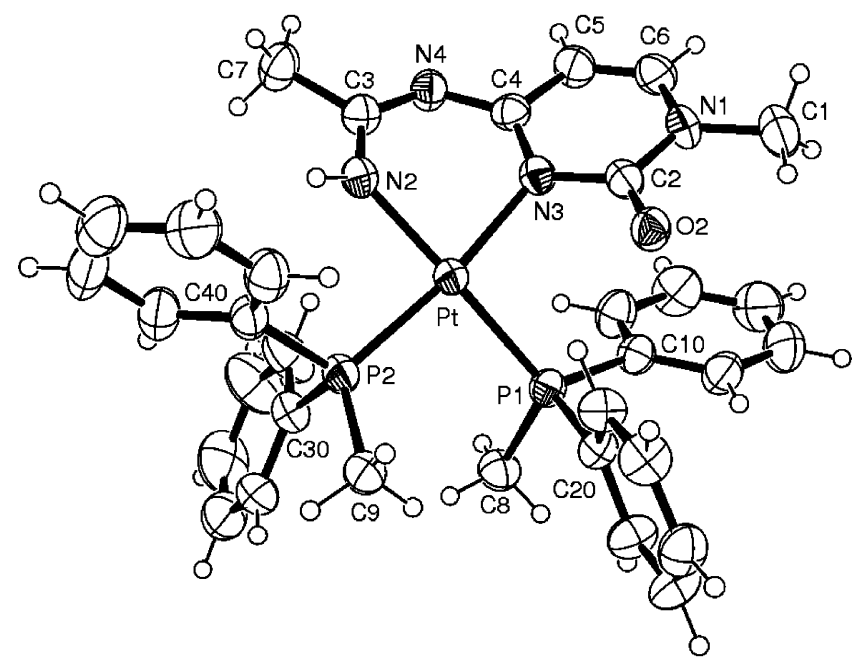

Figure 5. ORTEP drawing (ellipsoid 40\% probability level) of the cation of $5 \mathbf{a}$.

the cytosine ring. The overall complex conformation, as well as the bond distances, are close to those detected in the adenine derivatives, indicating a similar geometry in the fragment resulting from the binding of the acetonitrile molecule to the exocyclic amino nitrogen N(4). The perspective view of 5a (Figure 3 ) shows the orientation assumed by the chelating ligand with respect to the coordination plane (dihedral angle of $40.8(1)^{\circ}$ ).

The characterization of $\mathbf{5 a}, \mathbf{b}$ in solution was performed in $\mathrm{CD}_{3} \mathrm{CN}$, DMSO- $d_{6}$ and chlorinated solvents, and the pertinent ${ }^{1} \mathrm{H}$ NMR data are collected in Table 6.

The pyrimidinic protons occur as sharp doublets $\left({ }^{3} J_{\mathrm{HH}}=\right.$ $7.8 \mathrm{~Hz}$ ), and that attributed to $\mathrm{H}(5)$ shows a further splitting due to the coupling with a ${ }^{31} \mathrm{P}$ nucleus $\left({ }^{5} J_{\mathrm{HP}}=1.3-1.5 \mathrm{~Hz}\right)$. The NH proton in 5a exhibits a broad singlet (in DMSO$d_{6}$ ), while in $\mathbf{5 b}$ this signal has a fine structure (apparent triplet, $\left.{ }^{3} J_{\mathrm{HP}}=4-6\right) \mathrm{Hz}$ ) due to coupling with ${ }^{31} \mathrm{P}$ nuclei. This finding supports the protonation on the inserted acetonitrile nitrogen, as found in $\mathbf{3 a}$ and $\mathbf{3 b}$. Thus, the anionic ligand in $\mathbf{5} \mathbf{b}$ can be described as the deprotonated form of the amidine $N$-(1-methyl-2-oxo-2,3-dihydro- $1 H$-pyrimidin4-ylidene)-acetamidine (Chart 2).

The location of the $\mathrm{NH}$ proton in $\mathbf{5} \mathbf{a}$ remains unresolved. Its resonance, observed at room temperature as broad singlet at $\delta 6.29$ in DMSO- $d_{6}$, is shifted at $\delta 5.50$ and $5.53 \mathrm{ppm}$ in $\mathrm{CD}_{3} \mathrm{CN}$ and $\mathrm{CD}_{2} \mathrm{Cl}_{2}$, respectively, and attempts to observe a fine structure by lowering the temperature were unsuccessful. Moreover, whereas ${ }^{31} \mathrm{P}$ NMR spectra of $\mathbf{5 b}$ are characterized by a sharp $\mathrm{AB}$ multiplet, those of the $\mathrm{PMePh}_{2}$ analogue show that one part of the expected $\mathrm{AB}$ multiplet (at $\delta-8.0,{ }^{1} J_{\mathrm{PPt}}=3300 \mathrm{~Hz}$ ) is broad, in contrast with the sharp resonance at $\delta-6.5\left({ }^{1} J_{\mathrm{PPt}}=3300 \mathrm{~Hz}\right)$ as depicted in Figure 6 (trace a), in which the spectrum of a fresh solution of $5 \mathbf{a}$ in $\mathrm{CD}_{2} \mathrm{Cl}_{2}$ is reported.

As the temperature decreases, the spectrum becomes more complex, a series of changes leading to the appearance of two sets of resonances at $-40{ }^{\circ} \mathrm{C}$ (Figure 6, trace b). Similar temperature dependence and spectral patterns were observed in $\mathrm{CD}_{3} \mathrm{CN}$. In addition, in the $\left\{{ }^{1} \mathrm{H}\right\}{ }^{13} \mathrm{C}$ spectrum of $\mathbf{5 a}$ the 


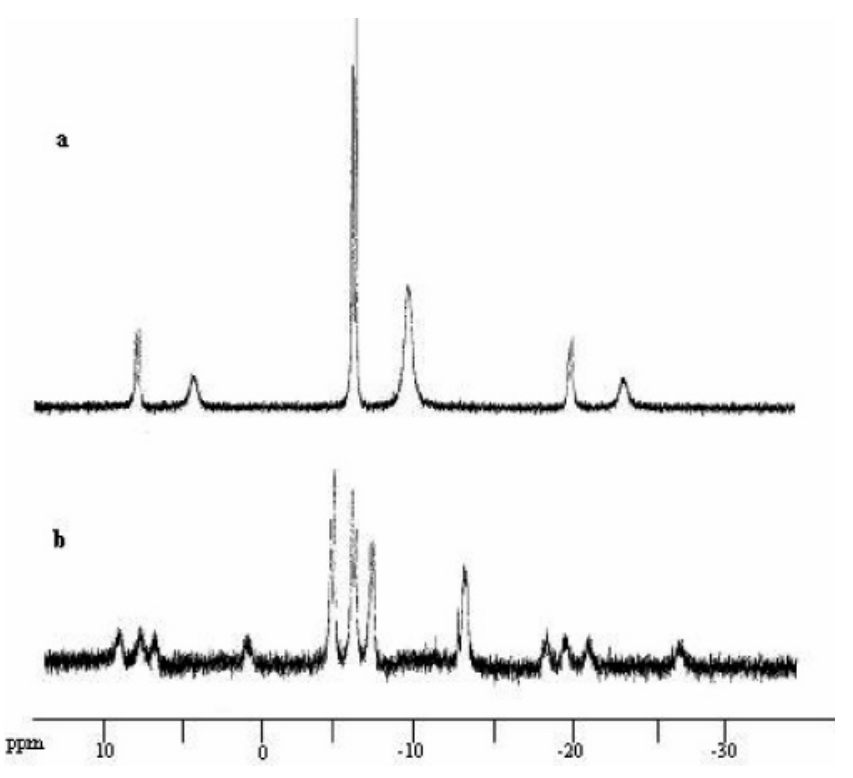

Figure 6. ${ }^{31} \mathrm{P}\left\{{ }^{1} \mathrm{H}\right\}$ NMR spectra of $\mathbf{5 a}$ in $\mathrm{CD}_{2} \mathrm{Cl}_{2}$ : (a) fresh solution at $27^{\circ} \mathrm{C}$; (b) at $-40{ }^{\circ} \mathrm{C}$.

resonances of one phosphine appear broad (phenyl region) or undetectable (methyl region), while in the proton spectrum the two phosphines exhibit distinct signals for methyl groups, one sharp (at $\delta 1.87$ with ${ }^{2} J_{\mathrm{HP}}=10.5 \mathrm{~Hz}$ and ${ }^{3} J_{\mathrm{HPt}}=36$ $\mathrm{Hz}$ ) and the other one broad (at $1.93 \mathrm{ppm}{ }^{2} J_{\mathrm{HP}}$ ca. $10 \mathrm{~Hz}$ with unresolved ${ }^{195} \mathrm{Pt}$ satellites).

These spectral features, not observed in the spectra of $\mathbf{3 a}$, are consistent with the presence of different conformations, related to restricted rotation of one phosphine around the $\mathrm{Pt}-\mathrm{P}$ bond in complex 5a and/or a different flexibility of the metallocycle. It is to be noted that in $\mathbf{3 a}$ the $\left\{{ }^{1} \mathrm{H}\right\}^{13} \mathrm{C}$ spectrum of the phenyl groups on the same phosphorus exhibits chemical equivalence (see experimental). This requires free rotation of phosphine ligands around the $\mathrm{Pt}-\mathrm{P}$ bonds and/or the presence of a symmetry plane which can be obtained if the six atom metallacycle and the adjacent purine ring are coplanar. From a detailed analysis of the crystal structures of the two complexes it is apparent that there is a stacking interaction between the cytosine and the $\mathrm{C}(10)$ phenyl ring in 5a (see Figure 5): the distance between their centroids is $3.86 \AA$, with torsion angle $\mathrm{N}(3)-\mathrm{Pt}-\mathrm{P}(1)-\mathrm{C}(10)$ of $-18.3^{\circ}$. The corresponding values in $\mathbf{3} \mathbf{a}$ are $4.20 \AA$ and $32.0^{\circ}(\mathrm{N}(1)-\mathrm{Pt}-\mathrm{P}(1)-\mathrm{C}(1 \mathrm{~b})$, respectively, with a tilting of the phenyl which does not provide a suitable stacking. It seems likely that the more bulkier carboxyl oxygen $\mathrm{O}(2)$ and methyl C(1) in the cytosine of 5a might hamper or reduce the free rotation in solution of the adjacent phosphine group, when compared with that of the $\mathrm{C}(2) \mathrm{H}$ environment in $\mathbf{3 a}$.

As shown for $\mathbf{3 a}, \mathbf{b}$, complexes $\mathbf{5 a}$ and $\mathbf{5 b}$ in solution are stable only in acetonitrile. 5a dissolved in a mixture of $\mathrm{CD}_{3} \mathrm{CN} / \mathrm{CH}_{3}{ }^{13} \mathrm{CN}$ slowly (several hours at room temperature) exchanges the inserted acetonitrile molecule, indicating a kinetic lability of the chelated ligand. ${ }^{22}$ Moreover, 5a and $\mathbf{5 b}$ in chlorinated solvents lose acetonitrile to give species not yet completely characterized.

Role of the Phosphine Ligands in the Activation of $\mathbf{C H}_{3} \mathbf{C N}$. Previous studies showed that cis- $\left[\left(\mathrm{PMe}_{3}\right)_{2} \mathrm{Pt}(\mu\right.$ -
Chart 3
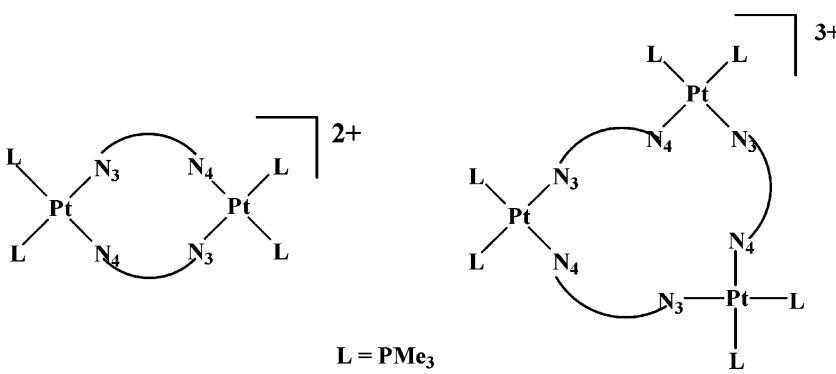

$\mathrm{OH}]_{2}\left(\mathrm{NO}_{3}\right)_{2}$, in water or DMSO, deprotonates the exocyclic $\mathrm{NH}_{2}$ group of $1-\mathrm{MeCy}$ affording the cyclic species cis$\left[\left(\mathrm{PMe}_{3}\right)_{2} \mathrm{Pt}\{1-\mathrm{MeCy}(-\mathrm{H})\}\right]_{2}\left(\mathrm{NO}_{3}\right)_{2}$ with the cytosinate ion bridging two metal centers through the $\mathrm{N}(3)$ and $\mathrm{N}(4)$ atoms (Chart 3). ${ }^{23}$ At $80{ }^{\circ} \mathrm{C}$, it converts quantitatively in the corresponding trinuclear derivative, cis- $\left[\left(\mathrm{PMe}_{3}\right)_{2} \mathrm{Pt}\{1-\mathrm{MeCy}-\right.$ $(-\mathrm{H})\}]_{3}\left(\mathrm{NO}_{3}\right)_{3}$, in which the nucleobase maintains the same coordination mode. ${ }^{24}$

Some of the polynuclear cyclic complexes, previously characterized, have been now prepared in acetonitrile and their stability in this solvent verified. We find that the perchlorate salt cis-[(PMe $\left.)_{2} \mathrm{Pt}\{9-\mathrm{MeAd}(-\mathrm{H})\}\right]_{2}\left(\mathrm{ClO}_{4}\right)_{2}$ (soluble in $\mathrm{CH}_{3} \mathrm{CN}$, unlike its nitrato derivative), ${ }^{7}$ can be prepared in good yield from cis- $\left[\left(\mathrm{PMe}_{3}\right)_{2} \mathrm{Pt}(\mu-\mathrm{OH})\right]_{2}\left(\mathrm{ClO}_{4}\right)_{2}$ and $9-\mathrm{MeAd}$ in acetonitrile, and it is indefinitely stable in this solvent, even at $50{ }^{\circ} \mathrm{C}$ for a week. A similar stability is exhibited by the trinuclear species cis-[(PMe $\left.2 \mathrm{Ph})_{2} \mathrm{Pt}\{9-\mathrm{MeAd}(-\mathrm{H})\}\right]_{3^{-}}$ $\left(\mathrm{NO}_{3}\right)_{3}$ and $c i s-\left[\left(\mathrm{PMe}_{2} \mathrm{Ph}\right)_{2} \mathrm{Pt}\{1-\mathrm{MeCy}(-\mathrm{H})\}\right]_{3}\left(\mathrm{NO}_{3}\right)_{3}$ which are quantitatively formed when $c i s-\left[\left(\mathrm{PMe}_{2} \mathrm{Ph}\right)_{2} \mathrm{Pt}(\mu-\mathrm{OH})\right]_{2}-$ $\left(\mathrm{NO}_{3}\right)_{2}$ reacts with 9-MeAd and 1-MeCy, respectively, in $\mathrm{CD}_{3} \mathrm{CN}^{10,13}$

The formation of the insertion products $\mathbf{3 a}, \mathbf{b}$ and $\mathbf{5 a}, \mathbf{b}$ seems therefore related to the presence of the $\mathrm{PMePh}_{2}$ and $\mathrm{PPh}_{3}$ ligands in the starting hydroxo complex. However, the relative importance of steric and/or electronic factors of the phosphines on the metal coordination of $\mathrm{CH}_{3} \mathrm{CN}$ and the following nucleophilic attack of the deprotonated nucleobase have to be elucidated. We observe that the trinuclear cation 2a can be isolated from acetonitrile while the formation of its $\mathrm{PPh}_{3}$ analogue is prevented, also in chlorinated solvents. As previously noticed, 2a in DMSO- $d_{6}$ undergoes a complete and rapid rearrangement with formation of the mononuclear cation $4 \mathbf{a}$ as the major component of the resulting mixture. ${ }^{5}$ On the contrary, in the same solvent, cis- $\left[\left(\mathrm{PMe}_{2} \mathrm{Ph}\right)_{2} \mathrm{Pt}\{9-\right.$ $\operatorname{MeAd}(-\mathrm{H})\}]_{3^{3+}}$ maintains almost completely its trinuclear structure. ${ }^{8}$ Similarly, the cytosine complex cis- $\left[\left(\mathrm{PMe}_{2} \mathrm{Ph}\right)_{2} \mathrm{Pt}-\right.$ $\{1-\mathrm{MeCy}(-\mathrm{H})\}]_{3}{ }^{3+}$ appears stable in DMSO, whereas we were unable to characterize similar species stabilized by $\mathrm{PMePh}_{2}$ and $\mathrm{PPh}_{3}{ }^{21}$

\section{Conclusions}

The formation of compounds $\mathbf{3}$ and $\mathbf{5}$ here described represents a rare example of a reaction in which a platinum-

(22) Wagner, G.; Pakhomova, T. B.; Bokack, N. A.; Fraùsto da Silva, J. J. R.; Vicente, J.; Pombeiro, A. J. L.; Kukushkin, V. Yu. Inorg. Chem. 2001, 40, 1683-1689.

(23) Trovò, G.; Bandoli, G.; Casellato, U.; Corain, B.; Nicolini, M.; Longato, B. Inorg. Chem. 1990, 29, 4616-4621.

(24) Schenetti, L.; Bandoli, G.; Dolmella, A.; Trovò, G.; Longato, B. Inorg. Chem. 1994, 33, 3169-3176. 


\section{Longato et al.}

nitrogen bond is formally added to a nitrile triple bond. The quality of the structural data obtained in the solid phase for these metallacycles does not allow us to discriminate between the azavinyledene complexes $c i s-\left[\mathrm{L}_{2} \mathrm{PtN}=\mathrm{C}(\mathrm{Me})\right.$ \{nucleobase$(-\mathrm{H})\}]^{+}$and the imino structures cis- $\left[\mathrm{L}_{2} \mathrm{PtNH}=\mathrm{C}(\mathrm{Me})-\right.$ $\{$ nucleobase $(-2 \mathrm{H})\}]^{+}$, in which the hydrogen on the $\mathrm{N}(6)$ (or $\mathrm{N}(4)$ ) atom of the $\mathrm{NH}_{2}$-deprotonated nucleobase is located on the nitrogen of the inserted $\mathrm{CH}_{3} \mathrm{CN}$ molecule. This latter structure appears to be only detectable on the solutions of $\mathbf{3 a}, \mathbf{b}$ and $\mathbf{5 b}$. The existence of a possible tautomeric equilibrium between the two forms seems also ruled out on the basis of a detailed analysis of the NMR spectra obtained in various solvents. This possibility, however, cannot be excluded for complex $\mathbf{5 a}$.

All these metallacycles are stable only in acetonitrile solution. In chlorinated solvents, 3a loses the inserted molecule of $\mathrm{CH}_{3} \mathrm{CN}$ to give the trinuclear species $\mathbf{2 a}$, whereas $\mathbf{3 b}$, having the bulkier $\mathrm{PPh}_{3}$ ligands, forms the mononuclear complex $\mathbf{4 b}$, stabilized by the chelation of the adenine at the $\mathrm{N}(6), \mathrm{N}(7)$ sites. For $\mathbf{2 a}$ and $\mathbf{4 b}$, the insertion of $\mathrm{CH}_{3} \mathrm{CN}$ into the platinum-nucleobase bond is a reversible reaction. On the contrary, for the cytosine derivatives $\mathbf{5 a}$ and $\mathbf{5 b}$, the relatively low stability of the trinuclear species $c i s-\left[\mathrm{L}_{2} \mathrm{Pt}\{1-\right.$ $\mathrm{MeCy}(-\mathrm{H})\}]_{3}{ }^{3+}$, when $\mathrm{L}$ is a $\mathrm{PMePh}_{2}$ and $\mathrm{PPh}_{3}$, make the loss of acetonitrile an irreversible process.

Acknowledgment. This work was financially supported by the Ministero dell'Universita' e della Ricerca Scientifica e Tecnologica, PRIN 2004. Thanks are due to Consorzio Interuniversitario di Ricerca in Chimica dei Metalli nei Sistemi Biologici for a Grant to D.M. We are in debt with Professor L. Schenetti for the assistance on the ${ }^{15} \mathrm{~N},{ }^{1} \mathrm{H}$ NMR experiments.

Supporting Information Available: Crystallographic data for the structures reported in this Article. This material is available free of charge via the Internet at http://pubs.acs.org.

IC051755F 